\title{
LIE ALGEBRAS OF CHARACTERISTIC $p$
}

\author{
BY \\ IRVING KAPLANSKY(1)
}

1. Introduction. Recent publications have exhibited an amazingly large number of simple Lie algebras of characteristic $p$. At this writing one cannot envisage a structure theory encompassing them all; perhaps it is not even sensible to seek one.

Seligman [3] picked out a subclass corresponding almost exactly to the simple Lie algebras of characteristic 0 . He postulated restrictedness and the possession of a nonsingular invariant form arising from a restricted representation. In this paper our main purpose is to weaken his hypotheses by omitting the assumption that the form arises from a representation. We find no new algebras for ranks one and two. On the other hand, it is known that new algebras of this kind do exist for rank three, and at that level the investigation will probably become more formidable.

For rank one we are able to prove more. Just on the assumption of a nonsingular invariant form we find only the usual three-dimensional algebra to be possible. Assuming simplicity and restrictedness permits in addition the survival of the Witt algebra. Still further information on algebras of rank one is provided by Theorems 1,2 and 4 .

Characteristics two and three are exceptions to nearly all the results. In those two cases we are sometimes able to prove more, sometimes less; for the reader's convenience, these theorems are assembled in an appendix. In addition, characteristic five is a (probably temporary) exception in Theorem 7.

TWO REMARKS ON STYLE. (a) Several proofs are broken up into a series of lemmas. To avoid endless repetition, these lemmas are stated in skeleton form, not intelligible beyond the immediate context. If, however, a lemma has an application occurring substantially later in the paper, its hypotheses are given in full.

(b) Binomial coefficients with subscript two abound in the paper. For the sake of typographical simplicity we adopt the unorthodox notation $\boldsymbol{n}_{2}$ $=n(n-1) / 2$. At one point we similarly write $n_{3}$ for $n(n-1)(n-2) / 6$.

\section{PART I. RANK ONE}

2. Basic definitions. We shall always be dealing with a finite-dimensional Lie algebra $L$ over an algebraically closed field of characteristic $p>0$. We use ordinary juxtaposition $x y$ for the operation in $L$; brackets are reserved for

Received by the editors February 7, 1957.

(1) Work on this paper was supported in part by the Office of Scientific Research of the Air Force. 
actual commutation in an associative algebra. We write $R_{x}$, rather than $\operatorname{Ad}(x)$, for the mapping $a \rightarrow a x$.

If $H$ is a nilpotent subalgebra of $L$, there is a unique decomposition $L$ $=\sum L_{\alpha}$ as a vector space sum; here each $\alpha$ is a scalar function on $H$ called a root, and $L_{\alpha}$ consists of all $x$ in $L$ which are annihilated by some power of $R_{h}-\alpha(h) I$ for every $h$ in $H$. We have $L_{\alpha} L_{\beta} \subset L_{\alpha+\beta}$ (in the sense that $L_{\alpha} L_{\beta}=0$ if $\alpha+\beta$ is not a root). Thus $L_{0}$ is a subalgebra containing $H$, and we say that $H$ is a Cartan subalgebra if $H=L_{0}$. An element $u$ is said to be regular if the multiplicity of the characteristic root 0 in $R_{u}$ is minimal; this minimal number is called the rank of $L$. If the above decomposition is performed with $H$ the one-dimensional subalgebra spanned by a regular element, it turns out that $L_{0}$ is a Cartan subalgebra. Thus there always exists a Cartan subalgebra whose dimension is the rank of $L$. One must beware of the fact that for characteristic $p>0$ the dimension of a Cartan subalgebra is not necessarily invariant; but of course the rank is a well defined invariant.

We shall consider restricted Lie algebras only when they are centerless. Thus we define a centerless Lie algebra $L$ to be restricted if the pth power of every inner derivation is inner. Then for every $x$ there exists a unique element $y$ satisfying $R_{y}=\left(R_{x}\right)^{p}$; we write $y=x^{p}$.

Let $L$ be a Lie algebra of rank one, and select any nonzero element $u$ in a one-dimensional Cartan subalgebra. Then the root spaces $L_{\alpha}$ are simply indexed by the characteristic roots of $R_{u}$ and are elements of the base field. By multiplying $u$ by a suitable scalar we can convert any desired root $\alpha$ into 1 .

Suppose $L$, in addition, is restricted (note that any Lie algebra of rank one is centerless). Then one easily sees that it is possible to choose $u$ so that $u^{p}=u$. It then follows that the roots are all in the prime field (integers mod $p$ ) and that $R_{u}$ acts as a scalar on each root space. Each of these facts makes for big simplifications.

The product of any element of $L_{\alpha}$ by an element of $L_{-\alpha}$ is a scalar multiple of $u$; this gives rise to an inner product between the spaces $L_{\alpha}$ and $L_{-\alpha}$. Of course this inner product is not necessarily nonsingular. Accordingly, we write $M_{\alpha}$ for the subspace of $L_{\alpha}$ annihilating $L_{-\alpha}$, and we have that the spaces $L_{\alpha} / M_{\alpha}$ and $L_{-\alpha} / M_{-\alpha}$ are paired in nonsingular fashion. We shall write $n(\alpha)$ for their common dimension. We shall prove ultimately (for characterstic $>3)$ that $n(\alpha)=0$ or 1 . In the next section we take a preliminary step.

3. Proof that $n(\alpha)$ is at most 2 . As we noted above, we may harmlessly assume that $\alpha=1$.

A simple product in a Lie algebra is one formed by successive multiplications by single elements. Write $N_{i}$ for the subspace spanned by all simple products of $i$ elements of $L_{1}$. Write $W_{i}$ for the subspace spanned by all simple products of $i$ elements of $L_{1}$, where at least one factor is in $M_{1}$ (the annihilator of $L_{-1}$ within $\left.L_{1}\right)$. Thus we have $W_{i} \subset N_{i} \subset L_{i}, N_{i+1}=N_{i} L_{1}, W_{i+1}$ $=N_{i} M_{1}+W_{i} L_{1}$. Note that $W_{1}=M_{1}, W_{2}=M_{1} L_{1}$. We also set $W_{0}=0, N_{0}=L_{0}$ ( $=$ the set of scalar muliples of $u$ ). 
Lemma $1 . N_{i}$ is invariant under $R_{u}$.

Proof. The lemma is clear for $i=1$, and we proceed by induction. By the Jacobi identity

$$
N_{i+1} u=\left(N_{i} L_{1}\right) u \subset\left(N_{i} u\right) L_{1}+N_{i}\left(L_{1} u\right) \subset N_{i} L_{1}+N_{i} L_{1}=N_{i+1} .
$$

Lemma $2 . W_{i}$ is invariant under $R_{u}$.

Proof. We first check this for $i=1$. We have to prove that $M_{1} u$ annihilates $L_{-1}$. By the Jacobi identity, $\left(M_{1} u\right) L_{-1} \subset\left(M_{1} L_{-1}\right) u+M_{1}\left(L_{-1} u\right)=0$. Then by Lemma 1 and induction:

$$
\begin{aligned}
W_{i+1} u & =\left(N_{i} M_{1}+W_{i} L_{1}\right) u \\
& \subset\left(N_{i} u\right) M_{1}+N_{i}\left(M_{1} u\right)+\left(W_{i} u\right) L_{1}+W_{i}\left(L_{1} u\right) \\
& \subset N_{i} M_{1}+W_{i} L_{1}=W_{i+1} .
\end{aligned}
$$

Lemma 3. $L_{-1} N_{i+1} \subset N_{i}$.

Proof. This is true for $i=0$ since $N_{1}=L_{1}, N_{0}=L_{0}$. Then by induction and Lemma 1,

$$
\begin{aligned}
L_{-1} N_{i+1} & =L_{-1}\left(N_{i} L_{1}\right) \subset\left(L_{-1} N_{i}\right) L_{1}+\left(L_{-1} L_{1}\right) N_{i} \\
& \subset N_{i-1} L_{1}+u N_{i}=N_{i} .
\end{aligned}
$$

Lemma 4. $L_{-1} W_{i+1} \subset W_{i}$.

Proof. This is true for $i=0$ since $W_{1}=M_{1}, W_{0}=0$. Then by induction and Lemmas 2 and 3:

$$
\begin{aligned}
L_{-1} W_{\mathrm{i}+1} & =L_{-1}\left(N_{i} M_{1}+W_{i} L_{1}\right) \\
& \subset\left(L_{-1} N_{i}\right) M_{1}+\left(L_{-1} M_{1}\right) N_{i}+\left(L_{-1} W_{i}\right) L_{1}+\left(L_{-1} L_{1}\right) W_{i} \\
& \subset N_{i-1} M_{1}+0+W_{i-1} L_{1}+u W_{i} \subset W_{i} .
\end{aligned}
$$

We insert at this point a lemma with several later applications.

Leмма 5. If $a, b$ are linearly independent elements of $L_{1}$ with a not in $M_{1}$ then $a b \neq 0$.

Proof. We can find $x \in L_{-1}$ with $a x=u$. We have $b \cdot a x+a \cdot x b+x \cdot b a=0$. Now $x b$ is a scalar multiple of $u$. If $a b=0$ we find that $b u$ is a scalar multiple of $a u$. Since $R_{u}$ is nonsingular on $L_{1}$, this is a contradiction.

Now let us'assume that $L_{1} / M_{1}$ is at least two-dimensional. Since $M_{1}$ is invariant under $R_{u}$ (Lemma 2) we may think of $R_{u}$ as acting on $L_{1} / M_{1}$ and pick a two-dimensional invariant subspace. Lifting a suitable basis of this back to $L_{1}$ we arrive at elements $a$ and $b$ which satisfy

$$
a u \equiv a, \quad b u \equiv b+\lambda a \quad\left(\bmod M_{1}\right)
$$

where $\lambda$ is a scalar (which we could take to be 0 or 1 ). Write $a b=c$. 
LEMMA 6. $c u \equiv 2 c\left(\bmod W_{2}\right)$.

Proof. By the Jacobi identity

$$
a b \cdot u+b u \cdot a+u a \cdot b=0 .
$$

If we make the replacements (1) in (2), and recall $W_{2}=M_{1} L_{1}$, we arrive at the statement in the lemma.

Since $a$ and $b$ are linearly independent modulo $M_{1}$, we can find an element $x$ in $L_{-1}$ such that $a x=u, b x=0$. Let $y$ be any element in $L_{-1}$ with $a y=0$. Write $a y=\nu u$ ( $\nu$ a scalar), and set $a u=d$.

LEMMA 7. $c x=-b u, c y=\nu d$.

Proof. These statements follow directly from the Jacobi identity applied to the triples $a, b, x$ and $a, b, y$ respectively.

LEMma 8. $d y=0$.

Proof. This follows from $a y=0$ and the fact that $a$ and $d$ differ by an element of $M_{1}$.

Define $c_{0}=c, c_{i}=c R_{d}^{i}$, and note that $c_{i}$ lies in $N_{i+2}$ for $i \geqq 1$.

LEMMA 9. $c_{i} y=0$ for $i \geqq 1$.

Proof. Apply the Jacobi identity to $c, d, y$, bearing Lemmas 7 and 8 in mind. The result is $c d \cdot y=0$, which is the case $i=1$ of the lemma. The general case then follows since $R_{d}$ and $R_{y}$ commute (Lemma 8).

LemMa $10 . c_{i} u \equiv(i+2) c_{i}\left(\bmod W_{i+2}\right)$.

Proof. The case $i=0$ is covered by Lemma 6 . We proceed by induction.

$$
c_{i} d \cdot u+d u \cdot c_{i}+u c_{i} \cdot d=0 .
$$

Now $d u \equiv d\left(\bmod M_{1}\right)$; this follows from the corresponding fact for $a$, the fact that $d$ and $a$ differ by an element of $M_{1}$, and the invariance of $M_{1}$ under $R_{u}$ (Lemma 2$)$. Hence $d u \cdot c_{i} \equiv d c_{i} \equiv-c_{i+1}\left(\bmod W_{i+3}\right)$. Applying our inductive assumption to $c_{i} u$ in (3) we arrive at $c_{i+1} u \equiv(i+3) c_{i+1}\left(\bmod W_{i+3}\right)$.

Lemma 11. $c_{1} x \equiv 3 c\left(\bmod W_{2}\right)$.

Proof. We write

$$
c d \cdot x+d x \cdot c+x c \cdot d=0 .
$$

Now $d x=u\left(\right.$ since $a x=u$ and $\left.a-d \in M_{1}\right)$, while $c u \equiv 2 c\left(\bmod W_{2}\right)$ by Lemma 6. Also $c x=-b u$ by Lemma 7 , and $b u \equiv b+\lambda a\left(\bmod W_{1}\right)$. Inserting these changes in (4), we obtain $t d \cdot x=c_{1} x \equiv 3 c\left(\bmod W_{2}\right)$.

Lemma 12. $c_{i} x \equiv(i+2)_{2} c_{i-1}\left(\bmod W_{i+1}\right)$.

Proof. The case $i=1$ is Lemma 11. Proceeding by induction and using 
Lemma 10, we find

$$
\begin{aligned}
c_{i+1} x & =c_{i} d \cdot x=-d x \cdot c_{i}-x c_{i} d \\
& \equiv-u c_{i}+(i+2){ }_{2} c_{i-1} d\left(\bmod W_{i+2}\right) \\
& \equiv(i+2) c_{i}+(i+2){ }_{2} c_{i}\left(\bmod W_{i+2}\right) \\
& =(i+3){ }_{2} c_{i} .
\end{aligned}
$$

Lemma 13. For $i \leqq p-3, c_{i}$ is not in $W_{i+2}$.

Proof. First, $c_{0}=c$ is not in $W_{2}$. For $c x=-b u$ (Lemma 7), and thus by Lemma 4 we would have $b u \in W_{1}=M_{1}$, a contradiction. We then apply Lemmas 4 and 12 , noting that the binomial coefficient $(i+2)_{2}$ is not 0 till $i=p-2$.

As a particular case of Lemma 13 we have that $c_{p-3}$ is not 0 . (Here we must insist on characteristic at least 5 . This assumption will be made henceforth tacitly in the lemmas, but it will be restated in the major theorems.) Now $y$ was taken to be any element of $L_{-1}$ annihilating $a$. Hence (Lemma 9) we have discovered a nonzero element $c_{p-3}$ in $L_{-1}$ which annihilates anything in $L_{-1}$ which annihilates $a$. Suppose now that $L_{-1} / M_{-1}$ has dimension $\geqq 3$. Then there are at least two elements of $L_{-1}$ linearly independent modulo $M_{-1}$ in the annihilator of $a$. Lemma 5 shows that $c_{p-3}$ must be a scalar multiple of each of these. This being impossible, we have proved:

LEMMA 14. $n(\alpha) \leqq 2$.

4. Preliminary results on the case $n(\alpha)=2$. We shall ultimately see that $n(\alpha)=2$ is impossible. In this section we collect a number of cases where $n(\alpha)=2$ turns out to be impossible. In $\S 6$ (with the aid of the results in $\S 5$ ) we shall complete the proof.

First we note that $n(2 \alpha)$ cannot vanish if $n(\alpha)=2$.

Lemma 15. If $n(\alpha)=2$, then $n(2 \alpha) \geqq 1$.

Proof. We assume $\alpha=1$. The spaces $L_{1} / M_{1}$ and $L_{-1} / M_{-1}$ are two-dimensional and are paired by a nonsingular inner product. We pick a basis for $L_{1} / M_{1}$ in Jordan canonical form under $R_{u}$, and then choose a dual basis for $L_{-1} / M_{-1}$. After lifting these bases to $L_{1}$ and $L_{-1}$ we obtain elements $a, b \in L_{1}, x, y \in L_{-1}$ satisfying $a x=b y=u, a y=b x=0, a u \equiv a, b u \equiv b+\lambda a$ $\left(\bmod M_{1}\right), \lambda=0$ or 1 . The equations $c x=-b u, c y=a u$ hold as in Lemma 7 , where $c$ is again $a b$. Then $c \cdot x y=-x \cdot y c-y \cdot c x=x \cdot a u+y \cdot b u=x a+y b=-2 u$. Thus $L_{2} L_{-2} \neq 0$ and $n(2) \geqq 1$.

We continue to assume $\alpha=1, n(1)=2$. Changing notation, we write $b_{1}, \cdots, b_{r}$ for a basis of $M_{1}$ and augment this basis by elements $a_{1}, a_{2}$ to a basis of $L_{1}$.

LеммA 16. The elements $a_{1} a_{2}, a_{1} b_{i}, a_{2} b_{j}$ are linearly independent $(1 \leqq i, j \leqq r)$. The dimension of $L_{2}$ is at least $2 r+1$. If $\operatorname{dim}\left(L_{2}\right) \leqq \operatorname{dim}\left(L_{1}\right)$, then $r=0$ or 1 . 
Proof. Assume

$$
\lambda a_{1} a_{2}+\sum \mu_{i} a_{1} b_{i}+\sum \nu_{j} a_{2} b_{j}=0 .
$$

We can find $x$ in $L_{-1}$ with $a_{1} x=u, a_{2} x=0$. Of course $b_{1} x=\cdots b_{r} x=0$. By the Jacobi identity,

$$
x \cdot a_{1} a_{2}=a_{2} u, \quad x \cdot a_{1} b_{i}=b_{i} u, \quad x \cdot a_{2} b_{j}=0 .
$$

Left-multiply (5) by $x$ and apply (6). Since $R_{u}$ is nonsingular on $L_{1}$, we obtain $\lambda=\mu_{i}=0$. Similarly $\nu_{j}=0$. The final statements of the lemma are evident.

Lemma 17. $\left(L_{-1} M_{-1}\right) L_{1}^{2}=0$.

Proof. Let $a \in L_{-1}, b \in M_{-1}, c, d \in L_{1}$. Then $b \cdot c d=0$ by the Jacobi identity on $b, c, d$. Also $b(a \cdot c d)=0$ since $a \cdot c d \in L_{1}$. An application of the Jacobi identity to $a, b$, and $c d$ then shows that $a b \cdot c d=0$.

Lemma 18. The following is impossible: $n(1)=n(2)=2$, and $L_{ \pm 1}, L_{ \pm 2}$ threedimensional.

Proof. From Lemma 16 we see that $L_{2}$ is spanned by $L_{1}^{2}$. Again by Lemma $5, L_{-1} M_{-1}$ is two-dimensional. This shows (by Lemma 17 ) that a two-dimensional subspace of $L_{-2}$ annihilates all of $L_{2}$. This is incompatible with the hypotheses that $n(2)=2$ and the spaces $L_{2}, L_{-2}$ are three-dimensional.

The next lemma will be used again in $\$ 5$ and $\S 11$; we accordingly state it in full with adequate generality.

LEMMA 19. Let L be a Lie algebra over an algebraically closed field of characteristic $p>3$. Let $\left\{L_{\alpha}\right\}$ denote the root spaces of $L$ under the decomposition relative to $R_{u}$, where $u \in L$. Assume $b \in L_{\alpha}, y \in L_{-\alpha}, t \in L_{-2 \alpha}, b y=u, y u=-\alpha y$, $t u=-2 \alpha t, t(b u-\alpha b)=0, t y=0$. Assume further that $t b \cdot b$ is a scalar multiple of $u$. Then $t=0$.

Proof. We may assume $\alpha=1$. Write $t b=t_{1}$. We apply the Jacobi identity three times: the triple $(t, b, y)$ yields $t_{1} y=-2 t$; the triple $(t, b, u)$ yields $t_{1} u$ $=-t_{1}$; the triple $\left(t_{1}, b, y\right)$ yields $t_{1} b \cdot y=-3 t_{1}$. Now $t_{1} b$ is a scalar multiple of $u$ by hypothesis. Hence $t_{1} b \cdot y$ is a scalar multiple of $y$. It follows that $t_{1} y=0$ and $t=0$.

Lemma 20. The following is impossible: $n(1)=2, L_{ \pm 1}$ two-dimensional, and $R_{u}$ acting as the identity on $L_{1}$.

Proof. $L_{1}$ and $L_{-1}$ are two-dimensional spaces paired by a nonsingular inner product. From the Jacobi identity, applied to $u, L_{1}$ and $L_{-1}$, we see that $R_{u}$ on $L_{-1}$ is the negative of the adjoint of $R_{u}$ on $L_{1}$. Thus $R_{u}=-I$ on $L_{-1}$. Choose dual bases: $a, b$ for $L_{1}$ and $x, y$ for $L_{-1}$. We now follow the notation and use the results in $\S 3$, defining $c=a b, c_{i}=c R_{a}^{4}$. It should be noted that in 
the present case $M_{1}=0$, so that the $W$ 's are all 0 and the congruences in $\S 3$ are replaced by equalities. Write $t=c_{p-4}$. By Lemma $9, t y=0$. By Lemma 10, $t u=-2 t$ (note that $t$ lies in $L_{-2}$ ). All the hypotheses of Lemma 19 are fulfilled (with $\alpha=1$ ) and we conclude that $t=0$. But this contradicts Lemma 13 .

Lemma 21. The following is impossible: $n(1)=2, \operatorname{dim}\left(L_{ \pm 1}\right)=2, \operatorname{dim}\left(L_{ \pm 2}\right)$ $=1$.

Proof. Since Lemma 20 rules out the possibility that $R_{u}$ is the identity on $L_{1}$, we have a basis $a, b$ for $L_{1}$ with $a u=a, b u=b+a$. On the dual basis $x, y$ for $L_{-1}$, we have $y u=-y, x u=-x-y$. Again we set $c=a b, c_{i}=c R_{a}^{i}$. Concerning the element $c_{p-4}$ we again have that it is a nonzero element in $L_{-2}$ and that it annihilates $y$. Now consider the element $x y$ in $L_{-2}$. The fact that $a c=c_{1}$ is not zero (Lemma 13) is mirrored by the fact that $x y$ does not annihilate $y$. Hence $x y$ and $c_{p-4}$ are linearly independent elements of $L_{-2}$, and $L_{-2}$ is at least two-dimensional.

Lemma 22. The following is impossible: $n(1)=n(2)=2, L_{ \pm 1}, L_{ \pm 2}$ all twodimensional.

Proof. We continue the analysis occurring in Lemma 21, studying further the linearly independent elements $c_{p-4}$ and $x y$ in $L_{-2}$. We have $x y \cdot u=-2 x y$; this is the analogue of the statement $c u=2 c$ (Lemma 6). Further, $c_{p-4} u$ $=-2 c_{p-4}$ by Lemma 10 . Since $L_{-2}$ is assumed to be two-dimensional, it is spanned by $x y$ and $c_{p-4}$; thus $R_{u}$ acts as $-2 I$ on $L_{-2}$. We can now invoke Lemma 20, with $L_{-2}$ playing the role of $L_{1}$.

Lemma 23. The following is impossible: $n(1)=n(2)=2, L_{1}$ two-dimensional, $L_{-1}$ and $L_{-2}$ three-dimensional.

Proof. Since $n(1)=2$ and $L_{-1}$ is three-dimensional, $M_{-1}$ is one-dimensional. Pick a nonzero element $z$ in $M_{-1}$. Choose a basis $a, b$ for $L_{1}$ as usual: $a u=a$, $b u=b+\lambda a$. The annihilator of $a$ in $L_{-1}$ will be a two-dimensional subspace containing $z$ which is invariant under $R_{u}$; thus it will have a basis $y, z$ satisfying $z u=-z, y u=-y+\mu z$. Now we repeat the idea of the last two lemmas, constructing the nonzero element $c_{p-4}$ in $L_{-2}$. This time $c_{p-4} y=c_{p-4} z=0$, for $c_{p-4}$ annihilates the annihilator of $a$ in $L_{-1}$ (Lemma 9). It follows that $c_{p-4} s$ $=0$, where $s=y z$. Now it is impossible for $c_{p-4}$ and $s$ to be linearly independent, for then by Lemma 5 they would both lie in $M_{-2}$, whereas $M_{-2}$ is onedimensional. Hence $c_{p-4}$ is a scalar multiple of $s$, and it follows that $y s=0$. Let $b$ be any element in $L_{1}$ with $b y=u$. Since $b z=0$, the Jacobi identity on $(b, y, z)$ yields $b s=z$. Applying the Jacobi identity to $(b, y, s)$ we obtain $s u=s$, which is impossible since $s \in L_{-2}$.

5. Study of the case $n(\alpha)=1$. In this section we begin to examine the case $n(\alpha)=1$. The results will be used in $\S 6$ to complete the proof that $n(\alpha)$ is at most one, and will again be used in $\$ 7$ to get our structure theorems. 
The preliminary lemmas have an application in $\$ 11$, and so we formulate them so as to apply to general Lie algebras (not necessarily of rank one).

Lemma 24. Let $a, x, u$ be elements in a Lie algebra $L$ with $a x=u$. Let $W$ be a subspace of $L$ which contains au-a, is invariant under $R_{u}$, and is annihilated by $R_{x}$. Let $T$ be a product (in any order) of $R_{a}{ }^{\prime} s, R_{x}{ }^{\prime} s, R_{u}$ 's and right multiplications by elements of $W$. Suppose $r$ of the factors are $R_{a}$ 's and $s$ are $R_{x}$ 's.

(a) If $s>r, W T=0$.

(b) If $s \leqq r$, assume in addition that $\left(W R_{a}^{k}\right) W=0$ for $0 \leqq k \leqq r-s$, and that at least one right-multiplication by an element of $W$ is actually present in $T$. Then again $W T=0$.

Proof. We shall prove this by a steady process of pushing terms $R_{u}, R_{x}$ to the left. Take the first factor in $T$ which is $R_{u}$ or $R_{x}$.

CASE I. It is $R_{u}$. If $T$ leads off with $R_{u}$, we can simply suppress $R_{u}$, for $W R_{u} \subset W$ by hypothesis. Otherwise there is a factor preceding $R_{u}$, and it is either $R_{a}$ or of the form $R_{d}$ with $d$ in $W$. Now

$$
R_{a} R_{u}=R_{u} R_{a}+R_{a}+R_{c}
$$

With each of the three terms on the right of (7), we have accomplished the desired progress of either eliminating $R_{u}$ or pushing it to the left. Again, $R_{d} R_{u}=R_{u} R_{d}+R_{e}, e \in W$, and the procedure is successful here too.

CASE II. It is $R_{x}$. If $T$ leads off with $R_{x}$, we have $W T=0$. Otherwise we have $R_{a}$ or $R_{d}$ preceding $R_{x}(d \in W)$. Now $R_{d}$ simply commutes with $R_{x}$, and for $R_{a} R_{x}$ we have the equation

$$
R_{a} R_{x}=R_{x} R_{a}+R_{u}
$$

In the case of the first term on the right of (8) we have pushed $R_{x}$ to the left. In the case of the second term we have eliminated one $R_{x}$ and one $R_{a}$, thereby maintaining the difference between the number of $R_{a}$ 's and the number of $R_{x}$ 's.

In case (a), we shall in this way eliminate all $R_{a}{ }^{\prime}$ s. At least one $R_{x}$ will always survive, and when it arrives at the extreme left, we get 0 .

In case (b), all $R_{x}$ 's and all $R_{u}$ 's get eliminated. What we have left is something of the form $R_{a}^{k} R_{e} \cdots$, where $e \in W$ and $0 \leqq k \leqq r-s$. (Note that the presence of at least one right-multiplication by an element of $W$ is preserved throughout the reduction process.) By hypothesis $W R_{a}^{k} R_{e}=0$. This concludes the proof of Lemma 24.

Lemma 25. Let $a, x, u$ be elements in a Lie algebra $L$ with $a x=u$. Let $W$ be a subspace of $L$ which contains au-a, is invariant under $R_{u}$, and is annihilated by $R_{x}$. Let an element $b$ in $W$, and integers $i, m, n(0 \leqq i, m \leqq n)$ be given. (a) If $m=n$, there is no further assumption. (b) If $m<n$, assume $\left(W R_{a}^{k}\right) W=0$ for $0 \leqq k \leqq n-m-1$. Then 


$$
b R_{a}^{i} R_{u} R_{a}^{n-i} R_{x}^{m}=b\left(R_{u}+i I\right) R_{a}^{n} R_{x}^{m} .
$$

where $I$ is the identity linear transformation.

Proof. We make an induction on $i$. For $i=0$, the equation is an identity. Assume (9) known for $i-1$ and apply (7). The term involving $R_{c}$ vanishes by Lemma 24 ; corresponding to the two cases of the present lemma we cite part (a) or (b) of Lemma 24. Hence

$$
\begin{aligned}
b R_{a}^{i} R_{u} R_{a}^{n-i} R_{x}^{m} & =b R_{a}^{i-1}\left(R_{u} R_{a}+R_{a}\right) R_{a}^{n-i} R_{x}^{m} \\
& =b\left[R_{u}+(i-1) I\right] R_{a}^{n} R_{x}^{m}+b R_{a}^{n} R_{x}^{m}
\end{aligned}
$$

be the inductive assumption. This proves the lemma.

Lemma 26. Let $a, x, u$ be elements in a Lie algebra $L$ with $a x=u$. Let $W$ be a subspace of $L$ which contains au $-a$, is invariant under $R_{u}$, and is annihilated by $R_{x}$. Let integers $m, n(0<m \leqq n)$ be given. (a) If $m=n$, there is no further assumption. (b) If $m<n$, assume $\left(W R_{a}^{k}\right) W=0$ for $0 \leqq k \leqq n-m-1$. Then for any $b$ in $W$,

$$
b R_{a}^{n} R_{x}^{m}=b T_{n-m+1} \cdots T_{n-1} T_{n} R_{a}^{n-m}
$$

where $T_{i}=i R_{u}+i_{2} I$.

Proof. First we prove, for any $i(0 \leqq i \leqq n)$,

$$
b R_{a}^{i} R_{x} R_{a}^{n-i} R_{x}^{m-1}=b T_{i} R_{a}^{n-1} R_{x}^{m-1} .
$$

For $i=0$, both sides of (11) vanish. Apply (8) on the left side of (11) to the first occurrence of $R_{a} R_{x}$, and then use induction and Lemma 25. The result is

$$
b R_{a}^{i} R_{x} R_{a}^{n-i} R_{x}^{m-1}=b\left[T_{i-1}+R_{u}+(i-1) I\right] R_{a}^{n-1} R_{x}^{m-1},
$$

which proves (11).

The special case $i=n$ of (11) reads

$$
b R_{a}^{n} R_{x}^{m}=b T_{n} R_{a}^{n-1} R_{x}^{m-1}
$$

By repeated use of (12) we obtain (10).

We now return specifically to the study of a Lie algebra $L$ of rank one. We shall assume in the remainder of this section that $n(1)=1$. Select an element $a$ in $L_{1}$ but not in $M_{1}$, and an element $x$ in $L_{-1}$ but not in $M_{-1}$; normalize so that $a x=u$. Since $M_{1}$ has co-dimension one in $L_{1}$ we have that $a u-a \in M_{1}$. Also, we recall (Lemma 2) that $M_{1}$ is invariant under $R_{u}$. Thus, with $W=M_{1}$, all the hypotheses of Lemmas 24-26, part (a), are fulfilled.

Lemma $27 . M_{1} R_{a}^{p-2} \subset M_{-1}$. 
Proof. Let $z$ be any element in $M_{-1}$. We have $a z=0, M_{1} z=0$. Hence $M_{1} R_{a}^{p-2}$ is annihilated by $z$. But $M_{1} R_{a}^{p-2} \subset L_{-1}$, and it follows from Lemma 5 that the annihilator of $M_{-1}$ within $L_{-1}$ is contained in $M_{-1}$. Hence $M_{1} R_{a}^{p-2}$ $\subset M_{-1}$.

For $j=1, \cdots, p-1$ set $S_{j}=M_{1} R_{a}^{j-1}$; thus $S_{1}=M_{1}$ and $S_{j} \subset L_{j}$. Write $S=S_{1}+\cdots+S_{p-1}$. Note that by Lemma $27, S_{p-1} a=0$, whence $S$ is invariant under $R_{a}$.

Lemma 28. $S_{1} S_{j}=0$.

Proof. We prove this by induction on $j$; the argument will simultaneously establish the initial stage $j=1$ of the induction.

Set $n=p-2, m=n-j+1$. If $j=1$, we have $m=n$, and Lemma 26 (a) is applicable. If $j>1$, we note that $n-m-1=j-2$. Our inductive assumption tells us then that $\left(W R_{a}^{\boldsymbol{k}}\right) W=0$ for $W=S_{1}$ and $0 \leqq k \leqq n-m-1$. Thus Lemma 26 (b) is applicable. The linear transformations $T_{n-m+1}, \cdots, T_{n}$ occurring on the right of (10) are easily seen to be nonsingular on $L_{1}$ and they map $S_{1}$ onto itself (since $S_{1}$ is invariant under $R_{u}$ ). We conclude that $R_{a}^{n} R_{x}^{m}$ induces a one-to-one linear transformation from $S_{1}$ onto $S_{1} R_{a}^{n-m}=S_{j}$. Now $S_{1}$ annihilates $S_{1} R_{a}^{n}$ (Lemma 27). Since $S_{1} x=0$, the annihilator of $S_{1}$ is stable under $R_{x}$. Hence $S_{1} S_{j}=S_{1}\left(S_{1} R_{a}^{n} R_{x}^{m}\right)=0$.

We record for later use the following facts (consequences of our observation that $R_{a}^{n} R_{x}^{m}$ induces a one-to-one linear transformation from $S_{1}$ onto $S_{j}$ ):

Lemma 29. The spaces $S_{1}, \cdots, S_{p-1}$ all have the same dimension. For $1 \leqq i \leqq p-2, R_{a}$ induces a one-to-one linear transformation from $S_{i}$ onto $S_{i+1}$. For $2 \leqq i \leqq p-1, R_{x}$ induces a one-to-one linear transformation from $S_{i}$ onto $S_{i-1}$.

Lemma $30 . S^{2}=0$.

Proof. We prove $S_{i} S=0$ by induction, noting that Lemma 28 is the case $i=1$. We have $S_{i} S=\left(S_{i-1} a\right) S \subset\left(S_{i-1} S\right) a+S_{i-1}(a S)=0$, since $a S \subset S$.

LEMma 31. $R_{u}$ acts as a scalar on each $S_{i}$.

Proof. We prove this first for $i=1$. Let $b$ be an element in $S_{1}$. By Lemma 27 we have $b R_{a}^{p-1}=0$. Apply Lemma 26 (a) with $m=n=p-1$. The linear transformations $T_{1}, \cdots, T_{p-1}$ which occur on the right of (10) are all nonsingular except the last, which is $R_{u}-I$. Hence $b\left(R_{u}-I\right)=0$.

Assume the lemma known for $i-1$. Then for $t \in S_{i-1}$ we have $t a \cdot u=t u \cdot a$ $+t \cdot a u$. Now $t u=(i-1) t$ by induction; $a u=a+c\left(c \in S_{1}\right)$ and $t c=0$ by Lemma 28. Hence $t a \cdot u=i \cdot t a$.

Lemma 32. Suppose that the dimension of $L_{i}$ is at most equal to the dimension of $L_{1}$. Then $S_{-i} L_{i}=0$.

Proof. Assume on the contrary that there exist elements $y \in S_{-i}, b \in L_{i}$ 
with $b y=u$. Now $S_{i}$ is a subspace of $L_{i}$ with the same dimension as $S_{1}$ (Lemma 29). Since $S_{1}$ is just one dimension short of $L_{1}$, it follows from our hypothesis that the codimension of $S_{i}$ in $L_{i}$ is 0 or 1 . In either event we have $b u-i b \in S_{i}$, since $S_{i}$ is invariant under $R_{u}$. Take any nonzero element $t$ in $S_{-2 i}$. Then $t y$ and $t(b u-i b)$ are both zero by Lemma 30 . Also, $t u=-2 i t, y u=-y$ by Lemma 31 . We have a contradiction of Lemma 19 (the notation is exactly that of Lemma 19, except that $\alpha$ is to be replaced by $i$ ).

6. Proof that $n(\alpha)$ is at most one. In this section we shall complete the proof that $n(\alpha)$ can never exceed one. We suppose that $n(\alpha)=2$ for some $\alpha$ and eventually reach a contradiction. Among all root spaces $L_{\alpha}$ with $n(\alpha)=2$ we may assume that $L_{1}$ has maximal dimension, and we write $r+2$ for that dimension. By Lemma $15, n(2)=1$ or 2 . We distinguish the two cases.

I. $n(2)=1$. By Lemma 16 , the dimension of $L_{2}$ is at least $2 r+1$. Thus $M_{2}$ (the annihilator of $L_{-2}$ within $L_{2}$ ) has a dimension (say $s$ ) which is at least $2 r$. The dimension of $L_{2}$ is $s+1$. We are now going to apply the theory of $\S 5$, with $L_{2}$ playing the role of the $L_{1}$ of that section. The sequence of $S_{i}$ 's there constructed arises anew here, but it starts from $S_{2}=M_{2}$, and we are principally concerned with the subspace of $L_{1}$ which emerges; the appropriate notation for it is $S_{1}$, but it is not to be confused with $M_{1}$ (the annihilator of $L_{-1}$ within $L_{1}$ ). Suppose that the dimension of $L_{1}$ is at most equal to that of $L_{2}$ (i.e., $r+2 \leqq s+1$ ). Then Lemma 32 applies to tell us that $S_{1} L_{-1}=0$, $S_{1} \subset M_{1}$. But $S_{1}$ is $s$-dimensional (Lemma 29) and $M_{1}$ is $r$-dimensional. Hence $s \leqq r$, a contradiction. Therefore the dimension of $L_{1}$ is strictly larger than that of $L_{2}$, that is, $r+1>s$. In conjunction with the inequality $s \geqq 2 r$, this implies $r=s=0$. Thus $L_{1}$ is two-dimensional, $L_{2}$ is one-dimensional. By our maximality assumption, $L_{-1}$ is also two-dimensional. By symmetry (we are now able to replace $L_{1}$ by $L_{-1}$ ), $L_{-2}$ is one-dimensional. But Lemma 21 asserts that this combination is impossible.

II. The demolition of Case I shows us that whenever $n(\alpha)$ is 2 , then $n(2 \alpha)$ is also 2. Moreover the dimension of $L_{2 \alpha}$ is at least that of $L_{\alpha}$; this is now clear if $L_{\alpha}$ is two-dimensional, and otherwise it follows from Lemma 16. But in fact the two dimensions are equal. For the chain of spaces $L_{\alpha}, L_{2 \alpha}$, $L_{4 \alpha}, \cdots$ closes back to $L_{\alpha}$, and so we must have equality at every step. Referring to Lemma 16 again, we see that the dimension of $L_{2 \alpha}$ must be 2 or 3 . Take $L_{1}$ to have $n(1)=2$ with (as above) maximal dimension among all $L_{\alpha}$ with $n(\alpha)=2$. Then there are three possibilities: (a) $\operatorname{dim}\left(L_{1}\right)=\operatorname{dim}\left(L_{-1}\right)$ $=2$, (b) $\operatorname{dim}\left(L_{1}\right)=3, \operatorname{dim}\left(L_{-1}\right) \doteq 2,(\mathrm{c}) \operatorname{dim}\left(L_{1}\right)=\operatorname{dim}\left(L_{-1}\right)=3$. In every case, $L_{2}$ has the same dimension as $L_{1}, L_{-2}$ the same as $L_{-1}$. We have prepared three lemmas exploding these three cases: Lemmas 22, 23, and 18, respectively.

We have completed the proof of our first major theorem.

THEOREM 1. Let L be a Lie algebra of rank one over an algebraically closed field of characteristic $>3$. Let $L_{\alpha}, L_{\beta}, \cdots$ be the root spaces relative to a one- 
dimensional Cartan subalgebra. Let $M_{\alpha}$ be the annihilator of $L_{-\alpha}$ within $L_{\alpha}$. Then: $L_{\alpha} / M_{\alpha}$ is either zero or one-dimensional.

7. Simple algebras with integral roots. In this section we shall determine the structure of a substantial class of simple Lie algebras of rank one.

THEOREM 2. Let $L$ be a simple Lie algebra of rank one over an algebraically closed field of characteristic $p>3$. Suppose that $L$ contains a regular element $u$ such that the characteristic roots of $R_{u}$ are in the prime field $G F(p)$. Then $L$ is either three-dimensional or isomorphic to the Witt algebra.

The proof will be given after several preliminary lemmas.

Among the root spaces $L_{i}(i=1, \cdots, p-1)$ for which $L_{i} L_{-i} \neq 0$ choose notation so that $L_{1}$ has maximal dimension. We adopt the notation and use the results of $\$ 5$.

LEMMA 33. $S_{-i} L_{i}=0$ for all $i$.

Proof. If $L_{i} L_{-i}=0$ there is nothing to prove. ()therwise, by our normalization to make $L_{1}$ have maximal dimension, the dimension of $L_{i}$ is at most that of $L_{1}$. Lemma 33 then follows from Lemma 32 .

Lemma 34. $S$ is an ideal in $L$.

Proof. By induction we prove simultaneously the following two statements

$$
\begin{aligned}
& S_{i} L_{j} \subset S \text { for } i+j \equiv t(\bmod p), \\
& L_{t} S \subset S .
\end{aligned}
$$

By symmetry it suffices to cover the range $t=0,1, \cdots,(p-1) / 2$. For $t=0$, (13) follows from Lemma 33, and (14) follows from Lemma 31. Suppose the two assertions are known for $t-1$. We proceed to prove (13) for the value $t$. The range $0 \leqq j \leqq t-1$ is already covered by our inductive assumption on (14). The case $j=t$ is vacuous, since $S_{0}=0$. The remaining range on $j$ is from $t+1$ to $p-1$; on $i$ the range is also from $t+1$ to $p-1$. We make an induction on $i$, noting that the induction can begin at $i=t$. Since $S_{i}=S_{i-1} a$ we have

$$
S_{i} L_{j} \subset a \cdot S_{i-1} L_{j}+S_{i-1} \cdot a L_{j} .
$$

The first term on the right of (15) lies in $S$ by our inductive assumption on $t$ in (13); the second lies in $S$ by our inductive assumption on $i$.

It remains to prove (14). That is, we must prove $L_{t} S_{k} \subset S$ for any $k$. The range $p-t \leqq k \leqq p-1$ is covered by our induction on (13). This leaves the range $1 \leqq k \leqq p-t-1$ to be covered. We make a descending induction on $k$, the induction beginning at $k=p-t$. Since $S_{k}=S_{k+1} x$ (Lemma 29), we have

$$
L_{t} S_{k} \subset L_{t} S_{k+1} \cdot x+L_{t} x \cdot S_{k+1} .
$$


The first term on the right of (16) lies in $S$ by our inductive assumption on $k$; the second lies in $S$ by our inductive assumption on $t$ in (14). This completes the proof of Lemma 34 .

If $L$ is simple, $S$ must be 0 (since, of course, $S \neq L$ ). We continue our analysis, assuming $S=0$. Thus $L_{1}$ and $L_{-1}$ are one-dimensional; we maintain our standard notation: $a \in L_{1}, x \in L_{-1}, a x=u$.

The next steps in the discussion are based on a computation like that occurring in $\S 5$. In the present context the work is much easier, simply because there is no term $R_{c}$ in (7). Since Lemmas 35-37 will be used again later we state them with adequate generality.

LemMA 35. Let $a, x, u, b$ be elements in a Lie algebra satisfying $a x=u, a u=a$, $b x \cdot a=0$. Then for any $n$

$$
b R_{a}^{n} R_{x}=b T_{n} R_{a}^{n-1}
$$

where $T_{n}=n R_{u}+n_{2} I$.

Proof. From $\left[R_{a} R_{u}\right]=R_{a}$ we first establish inductively:

$$
R_{a}^{n} R_{u}=\left(R_{u}+n I\right) R_{a}^{n} .
$$

Then we prove (17) by use of induction, (18), and (8).

Leмma 36. Let $L$ be a Lie algebra over an algebraically closed field of characteristic $p>3$. Let $a, x, u$ be elements of $L$ satisfying $a x=u, a u=a, x u=-x$. Let $\left\{L_{\alpha}\right\}$ denote the root spaces of $L$ under the decomposition induced by $R_{u}$ (note that $u$ is not assumed to be a regular element). Assume that $L_{0} R_{a}^{2} R_{x}$ and $L_{0} R_{x}^{2} R_{a}$ are 0 . Then: (a) $R_{a} R_{x}$ induces a one-to-one map of $L_{j}$ onto itself for $2 \leqq j \leqq(p-1) / 2$; (b) $R_{x} R_{a}$ induces a one-to-one map of $L_{j}$ onto itself for $(p+1) / 2$ $\leqq j \leqq p-2$.

Proof. By symmetry it is sufficient to prove (b). Suppose $b R_{x} R_{a}=0$, $b \in L_{j},(p+1) / 2 \leqq j \leqq p-2$. Write $m=p-j+2$. Then $b R_{a}^{m-2} \in L_{0}$, so that $b R_{a}^{m} R_{x}=0$. We note that $T_{n}=n R_{u}+n_{2} I$ is nonsingular on $L_{j}$ for $1 \leqq n \leqq m$. By repeated application of Lemma 35 we arrive at $b=0$.

The next lemma is an immediate consequence of Lemma 36.

Lemma 37. The hypotheses are the same as in Lemma 36. Then: for $2 \leqq j$ $\leqq(p-1) / 2, R_{a}$ maps $L_{j}$ one-to-one into $L_{j+1}$, and $R_{x}$ maps $L_{j+1}$ onto $L_{j}$. For $(p+1) / 2 \leqq j \leqq p-2, R_{a}$ maps $L_{j-1}$ onto $L_{j}$ and $R_{x}$ maps $L_{j}$ one-to-one into $L_{j-1}$.

We return to the context of simple Lie algebras of rank one. It is to be noted that the hypothesis $L_{0} R_{a}^{2} R_{x}=0$ of Lemmas 36 and 37 is fulfilled; indeed $u R_{a}^{2}=0$.

LEMMA 38. For $2 \leqq j \leqq(p-1) / 2, L_{j}$ is the direct sum of $L_{j-1} R_{a}$ and the null space of $R_{x} R_{a}$ in $L_{j}$. 
Proof. For $j=2$ this is clear since $R_{x} R_{a}$ annihilates $L_{2}\left(L_{2} R_{x} \subset L_{1}\right.$, and $L_{1}$ is the one-dimensional space spanned by $a$ ). Assume $j>2$. For $c \in L_{j}$, write $d=c x$. Since $R_{a} R_{x}$ is one-to-one on $L_{j-1}$ (Lemma 36), we may write $d=d_{1} R_{a} R_{x}$, $d_{1} \in L_{j-1}$. Then $c=\left(c-d_{1} a\right)+d_{1} a$ splits $c$ into a term annihilated by $R_{x}$ and one in $L_{j-1} R_{a}$. To see that the sum is direct, assume $c x=0, c=e a, e \in L_{j-1}$. Then $e a \cdot x=0$, whence $e=0$, since $R_{a} R_{x}$ is one-to-one on $L_{\jmath}$.

Lemma 39. For $2 \leqq j \leqq(p-1) / 2, R_{a}^{p-2 j}$ induces a one-to-one map of $L_{j}$ onto $L_{p-j}$, and $R_{x}^{p-2 j}$ induces a one-to-one map of $L_{p-j}$ onto $L_{j}$.

Proof. It will suffice to prove that $R_{a}^{p-2 j} R_{x}^{p-2 j}$ is one-to-one on $L_{j}$. Write $N_{i}$ for the null space of $R_{x} R_{a}$ in $L_{i}$. (Note that $N_{2}=L_{2}$, and that for $3 \leqq i$ $\leqq(p-1) / 2, N_{i}$ is by Lemma 37 simply the null space of $R_{x}$ in $\left.L_{i}\right)$. By iterated use of Lemma 38, we have that $L_{j}$ is the direct sum of $N_{j}, N_{j-1} R_{a}, \cdots, N_{2} R_{a}^{j-2}$. It will suffice to prove that $R_{a}^{p-2 j} R_{x}^{p-2 j}$ maps each $N_{i} R_{a}^{j-1}$ one-to-one onto itself $(2 \leqq i \leqq j)$. Now by iterated use of Lemma 35 we find, for $b$ in $N_{i}$,

$$
b R_{a}^{j-i} R_{a}^{p-2 j} R_{x}^{p-2 j}=b T_{j-i+1} T_{j-i+2} \cdots T_{p-j-i} R_{a}^{j-i} .
$$

For $n$ in the range from $j-i+1$ to $p-j-i$ we verify that $T_{n}=n R_{u}+n_{2} I$ is nonsingular on $L_{i}$. Also: $N_{i}$ is invariant under $R_{u}$ (and hence invariant under each $\left.T_{n}\right)$; for $i=2$ this is vacuous, while for $i>2$ it follows from $\left[R_{x} R_{u}\right]=-R_{x}$ and the fact that $N_{i}$ is merely the null space of $R_{x}$. Hence $R_{a}^{p-2 j} R_{x}^{p-2 j}$ induces a one-to-one mapping of $N_{i} R_{a}^{j-i}$ onto itself.

Lemma 40. Suppose $L_{r} L_{s}=0$, where $r=(p-1) / 2, s=(p+1) / 2$. Then: (a) $L_{i} L_{j}=0$ for $2 \leqq i, j \leqq p-2, i+j \equiv 1,0$ or $-1(\bmod p)$. (b) Either $x L_{2}$ or $a L_{-2}$ is 0 . (c) If $x L_{2}$ and a $L_{-2}$ are both 0 , then $L_{2}+L_{3}+\cdots+L_{p-2}$ is an ideal in $L$.

Proof. (a). By symmetry we can confine ourselves to the cases $i+j \equiv 1$ or $0(\bmod p)$. We can, moreover, confine $j$ to the range $s, \cdots, p-2$, and then $i$ will be either $p-j$ or $p+1-j$. We make an ascending induction on $j$. To start the induction at $j=s$, we have to check $L_{r} L_{s}=L_{s} L_{s}=0$. The first of these is zero by hypothesis. To prove $L_{s} L_{s}=0$, it is sufficient to show that it annihilates $x$. Since $L_{8} x \subset L_{r}$, this follows from the Jacobi identity.

Suppose now that $L_{i} L_{j}=0$ is known for a certain $j$, where $i$ is either $p-j$ or $p+1-j$. We proceed to $j+1$. We are supposing of course that $j+1$ is at most $p-2$. Hence Lemma 37 is applicable and tells us that $L_{j+1}$ is equal to $L_{j} a$. Then

$$
L_{i} L_{j+1} \subset a \cdot L_{i} L_{j}+a L_{i} \cdot L_{j} \subset a \cdot L_{i} L_{j}+L_{i+1} L_{j} .
$$

Take $i=(p+1)-(j+1)=p-j$. Then the right side of (19) vanishes by our induction on $j$. The other value of $i$ we have to treat is $i=p-(j+1)$. Since $j+1 \leqq p-2$, we have $i \geqq 2$. Hence $L_{i}=L_{i+1} x$ by Lemma 37 . Then 


$$
L_{i} L_{j+1} \subset x \cdot L_{i+1} L_{j+1}+L_{i+1} L_{j} .
$$

The first term on the right of (20) vanishes by the case we have just treated. The second vanishes by our induction on $j$.

(b) Suppose for instance that $L_{-2} a$ is not zero (and hence equal to $L_{-1}$ ). We prove that $L_{2} x$ vanishes by pushing the first half of the argument of part (a) one step further. In detail:

$$
L_{2} L_{-1}=L_{2} \cdot L_{-2} a \subset a \cdot L_{2} L_{-2}+L_{3} L_{-2},
$$

which vanishes by part (a).

(c) If $L_{2} x=L_{-2} a=0$, then $L_{2}+\cdots+L_{p-2}$ admits multiplication by $L_{1}$ and $L_{-1}$ (as well as by $L_{0}$ of course). By part (a), $L_{2}+\cdots+L_{p-2}$ is a subalgebra. Hence it is an ideal.

We are ready to proceed to the proof of Theorem 2 itself. By Lemma 37, the spaces $L_{2}, \cdots, L_{r}(r=(p-1) / 2)$ have dimensions which increase, in the weak sense. The same is true for the sequence of spaces $L_{p-2}, \cdots, L_{s}$ $(s=(p+1) / 2)$. The spaces $L_{r}$ and $L_{s}$ have the same dimension. If that common dimension is 0 or 1 , then all $L_{i}$ have dimension 0 or 1 . In this case Zassenhaus [4, pp. 37-47] has proved that $L$ is either 3-dimensional or the Witt algebra. We therefore assume henceforth that $L_{r}$ and $L_{s}$ have dimension greater than one and shall eventually reach a contradiction. We have that $L_{r} L_{s}=0$, for otherwise Lemma 34 shows that $L$ has a nontrivial ideal. (Alternatively, we could cite again our initial normalization of $L_{1}$ to have maximal dimension among all spaces $L_{i}$ with $L_{i} L_{-i} \neq 0$ ). We apply parts (b) and (c) of Lemma 40. For definiteness let us assume $L_{2} x \neq 0, L_{-2} a=0$. Select $d \in L_{2}$ with $d x=a$.

Write $Z_{i}=T_{1}+\cdots+T_{i}$, where, as always, $T_{n}=n R_{u}+n_{2} I$. One computes that $Z_{i}=(i+1)_{2} R_{u}+(i+1)_{3} I$. We next prove that for any element $b$ with $b x=0$, and any $j$,

$$
b R_{a}^{j} R_{d} R_{x}^{j+2}=b Z_{j+1} T_{1} \cdots T_{j} .
$$

For $j=0,(21)$ is verified by applying $\left[R_{d} R_{x}\right]=R_{a}$ and (17) with $n=1$. We assume (21) known for $j-1$. Then

$$
\begin{aligned}
b R_{a}^{j} R_{d} R_{x}^{j+2} & =b R_{a}^{j}\left(R_{x} R_{d}+R_{a}\right) R_{x}^{j+1} \\
& =b T_{j} R_{a}^{j-1} R_{d} R_{x}^{j+1}+b R_{a}^{j+1} R_{x}^{j+1} \\
& =b T_{j} Z_{j} T_{1} \cdots T_{j-1}+b T_{1} T_{2} \cdots T_{j+1} \\
& =b Z_{j+1} T_{1} \cdots T_{j} .
\end{aligned}
$$

(In this computation we used $b T_{j} R_{x}=0$, which follows from $b x=0$ and $\left[R_{x} R_{u}\right]=-R_{x}$.)

In the sequence of spaces $L_{1}, L_{2}, \cdots, L_{r}$ the first is one-dimensional and the last has dimension greater than one. $R_{x}$ maps each into its predecessor. 
Hence for some $k$ with $2 \leqq k \leqq r=(p-1) / 2$ there exists a nonzero element $b$ in $L_{k}$ with $b x=0$. Apply (21) with $j=p-2 k-1$. It is readily computed that $T_{1}, \cdots, T_{j}, Z_{j+1}$ are all nonsingular on $L_{k}$, and thus the right side of (21) is nonzero. We now distinguish two cases.

I. $k=2$. Then $b R_{a}^{j} R_{d}$ lies in $L_{p-1}$ and is annihilated by $R_{x}$, a contradiction.

II. $k>2$. Write $c=b R_{a}^{j} R_{d}$. We note that the right side of (21) is annihilated by $R_{x}$, for the null space of $R_{x}$ is invariant under $R_{u}$ and hence under all the $T$ 's and $Z$ 's. Hence $c R_{x}^{j+3}=0$. Now $c$ lies in $L_{p-k+1}$ and we have $s$ $\leqq p-k+1 \leqq p-2$. Also, $j+3=p-2 k+2$. But by Lemma $39, R_{x}^{p-2 k+2}$ is oneto-one on $L_{p-k+1}$. Hence $c=0$, a contradiction of the fact that the right side of (21) is nonzero. This completes the proof of Theorem 2.

8. Restricted algebras. If $L$ is a restricted simple Lie algebra of rank one, the hypotheses of Theorem 2 are fulfilled, as we noted in $\$ 2$. Hence:

THEOREM 3. Let $L$ be a restricted simple Lie algebra of rank one over an algebraically closed field of characteristic $p>3$. Then $L$ is either three-dimensional or isomorphic to the Witt algebra.

For the reader who wishes to reach this theorem rapidly, we remark that large portions of the preceding material can be by-passed. The crucial parts are: Lemma 19, Lemma 20 , the latter half of $\S 5$, and $\S 7$.

9. Algebras with an invariant form. An invariant form on a Lie algebra $L$ is a symmetric bilinear form $f$ satisfying $f(a b, c)=f(a, b c)$ for all $a, b, c$ in $L$. It is nonsingular if no nonzero element of $L$ is orthogonal to all of $L$. It then follows that any Cartan subalgebra $H$ is also nonsingular relative to $f$, and that $L_{\alpha}, L_{-\alpha}$ are nonsingular dual spaces under $f$ (see $[3$, p. 8]). From this we can further deduce that no nonzero element $x$ of $L_{\alpha}$ can annihilate all of $L_{-\alpha} ; x L_{-\alpha}=0$ implies $f\left(x L_{-\alpha}, H\right)=0, f\left(x, L_{-\alpha} H\right)=0, x=0$, since $L_{-\alpha} H=L_{-\alpha}$.

Let us analyze the algebras of rank one possessing this last property.

THEOREM 4. Let $L$ be a Lie algebra of rank one over an algebraically closed field of characteristic $p>3$. Assume that no nonzero element of a root space $L_{\alpha}$ annihilates all of $L_{-\alpha}$. Then: all root spaces are one-dimensional. If $L$ is not three-dimensional, the roots form a group under addition.

Proof. That the root spaces are all one-dimensional follows at once from Theorem 1.

Suppose that $L$ is not three-dimensional. Let $\alpha$ and $\beta$ be roots; we must show that $\beta-\alpha$ is a root. We do this first on the assumption that $\beta$ is not an integral multiple of $\alpha$. Normalize so that $\alpha=1$. Pick nonzero elements $a$ in $L_{1}, x$ in $L_{-1}, b$ in $L_{\alpha}$, normalized so that $a x=u$. If $\alpha-1$ is not a root, $b x=0$. Lemma 35 is applicable and shows in particular that $b R^{p-1}$ is not 0 . But then $\alpha-1$ is a root after all.

What remains to be shown is this: if $L$ is not three dimensional and 1 is a root, then $i$ is a root for every $i$ in $G F(p)$. Let $M$ denote the subalgebra $\sum L_{i}$, 
$i \in G F(p) . M$ is easily seen to be simple, and if it is not three-dimensional, it is the Witt algebra by Theorem 2. In particular, every $i$ in $G F(p)$ is a root. We shall prove that $M$ is not three dimensional. If it is, there is some root $\alpha$ not in $G F(p)$. By the result proved in the preceding paragraph, $\alpha-1$ and $\alpha+1$ are roots. Moreover, since $\alpha+1$ is not an integral multiple of $\alpha-1$, $(\alpha+1)-(\alpha-1)=2$ is a root. This contradiction completes the proof of Theorem 4 .

THEOREM 5. Let $L$ be a Lie algebra of rank one over an algebraically closed field of characteristic $p>3$. Assume that $L$ possesses a nonsingular invariant form $f$. Then $L$ is three-dimensional.

Proof. We continue where Theorem 4 leaves off. Assume that $L$ is not three-dimensional and normalize so that 1 is a root. Pick $a, b$ nonzero elements in $L_{1}, L_{(p-1) / 2}$ respectively. We have $f(a b, b)=f(a, b b)=0$. Since $f$ is nonsingular between $L_{(p-1) / 2}$ and $L_{(p+1) / 2}$ this implies $a b=0$, contradicting Lemma 37 (what we have just shown is merely that, for $p>3$, the Witt algebra does not admit a nonsingular invariant form).

\section{PART II. RANK Two}

10. The diagonable case. In Part II we shall be dealing mainly with a Lie algebra $L$ over an algebraically closed field of characteristic $p>3$, which is centerless, restricted, and possesses a nonsingular invariant form. If $H$ is a Cartan subalgebra of $L$, it is easily seen that $h \in H$ implies $h^{p} \in H$. If $H$ is twodimensional it follows from the results in [1] that there is a basis $u, v$ for $H$ with $u^{p}=u, v^{p}=v$ or 0 . We study the two cases separately.

If $u^{p}=u, v^{p}=v$ then $H$ acts diagonally on $L$. More generally, assume that $L$ is any Lie algebra with a nonsingular invariant form $f$ and a Cartan subalgebra $H$ that acts diagonally. For any root $\alpha$ there is a unique element $h_{\alpha} \in H$ such that $\alpha(h)=f\left(h, h_{\alpha}\right)$ for every $h \in H$. We call $\alpha$ nonisotropic if $f\left(h_{\alpha}, h_{\alpha}\right) \neq 0$.

THEOREM 6. Let $L$ be a centerless Lie algebra over an algebraically closed field of characteristic $p>3$. Assume that $L$ possesses a nonsingular invariant form $f$ and a Cartan subalgebra that acts diagonally. Then: all roots in $L$ are nonisotropic.

Proof. We assume that $\alpha$ is an isotropic root and eventually reach a contradiction. Call a root $\beta$ orthogonal to $\alpha$ if $f\left(h_{\alpha}, h_{\beta}\right)=0$.

(a) If $\beta$ is not orthogonal to $\alpha$, then $\beta-\alpha$ is a root.

Pick $a \in L_{\alpha}, x \in L_{-\alpha}$ with $f(a, x)=-1$. Then $a x=h_{\alpha}$ [3, Corollary 3.2]. Since $\alpha$ is isotropic, $a h_{\alpha}=x h_{\alpha}=0$. Take $b \neq 0$ in $L_{\beta}$. Assume that $\beta-\alpha$ is not a root; then $b x=0$. We now prove

$$
b R_{a}^{i} R_{x}=i \beta\left(h_{\alpha}\right) b R_{a}^{i-1} .
$$


For $i=0$, both sides vanish. Assume (22) known for $i-1$. Then

$$
\begin{aligned}
b R_{a}^{i} R_{x} & =b R_{a}^{i-1}\left(R_{x} R_{a}+R_{h_{\alpha}}\right) \\
& =(i-1) \beta\left(h_{\alpha}\right) b R_{a}^{i}+b R_{h_{\alpha}} R_{a}^{i-1}
\end{aligned}
$$

since $R_{a}$ and $R_{h_{\alpha}}$ commute. Since $b \in L_{\beta}$ and $A$ acts diagonally, $b h_{\alpha}=\beta\left(h_{\alpha}\right) b$. Thus (22) is proved. The element $b R_{a}^{p-1}$ vanishes, for $\beta-\alpha$ is not a root. By iterated use of (22) we reach the contradiction $b=0$ (note that $\beta\left(h_{\alpha}\right)$ is nonzero by hypothesis).

(b) If $\gamma$ is a nonisotropic root, $2 \gamma$ is not a root.

Form the algebra $M$ spanned by $h_{\gamma}$ and $\sum L_{i \gamma}, i$ running over all nonzero integers $\bmod p . M$ is an algebra of rank one with a nonsingular invariant for $\mathrm{m}$. By Theorem $5,2 \gamma$ is not a root.

(c) If $\beta$ is another isotropic root, then $\beta$ is orthogonal to $\alpha$.

Suppose on the contrary that $\beta$ is not orthogonal to $\alpha$. By part (a), $\alpha+\beta$ is a root. Since $f\left(h_{\alpha}, h_{\alpha+\beta}\right)=f\left(h_{\alpha}, h_{\alpha}+h_{\beta}\right)=f\left(h_{\alpha}, h_{\beta}\right) \neq 0, \alpha+\beta$ is not orthogonal to $\alpha$. Hence, again by (a), $2 \alpha+\beta$ is a root. Similarly we argue that $2 \alpha+\beta$ is not orthogonal to $\beta$, and hence $2 \alpha+2 \beta$ is a root. But $f\left(h_{\alpha+\beta}, h_{\alpha+\beta}\right)=2 f\left(h_{\alpha}, h_{\beta}\right)$ $\neq 0$. Thus $\alpha+\beta$ is a nonisotropic root. We have contradicted part (b).

(d) If $\gamma$ is not orthogonal to $\alpha$ (hence, by (c), is nonisotropic), then $f\left(h_{\alpha}, h_{\gamma}\right) / f\left(h_{\gamma}, h_{\gamma}\right)$ cannot be an integer.

Suppose this ratio is an integer $i$, necessarily different from 0 . Let $j$ $=-(2 i)^{-1}$. By iterated use of (a), $\delta=\gamma+j \alpha$ is a root. However $f\left(h_{\delta}, h_{\delta}\right)$ $=f\left(h_{\gamma}+j h_{\alpha}, h_{\gamma}+j h_{\alpha}\right)=0, f\left(h_{\delta}, h_{\alpha}\right) \neq 0$. This contradicts (c).

(e) The proof of (d) shows further: if $\gamma$ is not orthogonal to $\alpha$, each $\gamma+i \alpha$ ( $i$ an integer) is a nonisotropic root.

(f) We now complete the proof of Theorem 6. There must exist some root $\gamma$ which is not orthogonal to $\alpha$; otherwise $h_{\alpha}$ would be central, contrary to hypothesis. By (e), $\alpha-\gamma$ and $\alpha+\gamma$ are nonisotropic roots. We claim that $\alpha-2 \gamma$ is not a root. If it were, $\alpha-2 \gamma+\alpha=2(\alpha-\gamma)$ would also be a root by (a), contrary to (b). Similarly $\alpha+2 \gamma$ is not a root. Write $u=h_{\gamma} / f\left(h_{\gamma}, h_{\gamma}\right)$. Select $a \in L_{\gamma}, x \in L_{-\gamma}$ with $a x=u$; note that $a u=a, x u=-x$. Take $b \neq 0$ in $L_{\alpha-\gamma}$. We have $b x=0, b R_{a}^{3}=0$. Let $n$ be the smallest integer such that $b R_{a}^{n}$ $=0(1 \leqq n \leqq 3)$. Lemma 35 is applicable and tells us that $b T_{n}=b\left(n R_{u}+n_{2} I\right)$ $=0$. Since $b h_{\gamma}=f\left(h_{\alpha-\gamma}, h_{\gamma}\right) b$, we deduce that $f\left(h_{\alpha-\gamma}, h_{\gamma}\right)+(n-1) f\left(h_{\gamma}, h_{\gamma}\right)=0$. It follows that $f\left(h_{\alpha}, h_{\gamma}\right) / f\left(h_{\gamma}, h_{\gamma}\right)$ is an integer, in contradiction to (d).

Theorem 6 is a generalization of Theorem 4.2 in [3]. Since Seligman makes no further use of the assumption that his invariant form arises from a representation, this accomplishes the classification of simple restricted Lie algebras with a nonsingular invariant form and a Cartan subalgebra that acts diagonally.

11. The nondiagonable case. It remains to discuss simple restricted Lie algebras of rank two with a nonsingular invariant form and a Cartan sub- 
algebra with basis $u$, v satisfying $u^{p}=u, v^{p}=0$. In this section we shall show that such algebras do not exist.

THEOREM 7. Let $L$ be a restricted simple Lie algebra of rank two over an algebraically closed field of characteristic $>5$. Assume that $L$ possesses a nonsingular invariant form. Then any two-dimensional Cartan subalgebra of $L$ acts diagonally.

The proof will be broken up into a number of lemmas.

On any root space we have $R_{0}^{p}=0$. Hence the roots are indexed merely by the characteristic roots of $R_{u}$ : the integers $\bmod p$. On $L_{i}, R_{u}$ acts as $i$ times the identity.

Lemma 41. If $a \in L_{1}$ and $a v \neq 0$, then $a^{p}=0$.

Proof. It is easily seen that $a^{p}$ lies in $H$. Moreover $a \cdot a^{p}=0$. If $a v \neq 0$, no nonzero element of $H$ annihilates $a$.

We shall make a large number of computations of a product of an element $a$ in $L_{1}$ by an element $x$ in $L_{-1}$ in the following way: to determine $a x$ it suffices to know $f(a x, u)$ and $f(a x, v)$, since $f$ is nonsingular on $H$. Now $f(a x, u)=f(a, x u)$ $=-f(a, x) ; f(a x, v)=-f(a v, x)$. So the ingredients of the computation are provided by the inner products of $x$ with $a$ and $a v$. A large number of cases are covered by the next lemma; when slightly different computations have to be made later we shall leave them to the reader.

Lemma 42. Suppose that $a_{i} \in L_{1}, x_{i} \in L_{-1}(i=1, \cdots, n)$; that $a_{i} v=a_{i+1}$ $(i=1, \cdots, n-1)$; and that $f\left(a_{i}, x_{j}\right)=\delta_{i j}$ (Kronecker delta). Then: (a) $a_{1} x_{1}$ $=\cdots=a_{n} x_{n}$. This element is orthogonal to $v$ and has inner product -1 with $u$. (b) $a_{1} x_{2}=a_{2} x_{3}=\cdots=a_{n-1} x_{n}$. This element is orthogonal to $u$ and has inner product -1 with $v$. (c) $a_{i} x_{j}=0$ if $j \neq i$ or $i+1$.

Proof. (a) $f\left(a_{i} x_{i}, u\right)=-1$ and $f\left(a_{i} x_{i}, v\right)=-f\left(a_{i+1}, x_{i}\right)=0$.

(b) $f\left(a_{i} x_{i+1}, u\right)=0$ and $f\left(a_{i} x_{i+1}, v\right)=-f\left(a_{i+1}, x_{i+1}\right)=-1$.

(c) If $j \neq i$ or $i+1, a_{i} x_{j}$ is orthogonal to both $u$ and $v$.

Lemma 43. If $a \in L_{1}, a v \neq 0$, then $a L_{-1}$ is all of $H$.

Proof. The elements $a_{1}=a, a_{2}=a v$ are linearly independent. Therefore we can find elements $x_{1}, x_{2}$ in $L_{-1}$ such that $f\left(a_{i}, x_{j}\right)=\delta_{i j}$. By Lemma $42, a_{1} x_{1}$ is nonzero and orthogonal to $v ; a_{1} x_{2}$ is nonzero and orthogonal to $u$. Hence $a_{1} x_{1}$ and $a_{1} x_{2}$ span $H$.

Lemma 44. For any $a \in L_{1}$ we have (av) $R_{a}^{p-2}=0$.

Proof. If $a v=0$ there is nothing to prove. Otherwise by Lemma 43 there exists $z \in L_{-1}$ with $a z=v$. By Lemma $41, z R_{a}^{p}=0$. Since $z R_{a}^{2}=v a$, this proves the lemma.

Lemma 45. $f(v, v)=0$. 
Proof. We must have $R_{v} \neq 0$; otherwise $v$ is in the center of $L$. Thus we may assume the existence of an element $a$ in $L_{1}$ with $b=a v \neq 0$. Let us write $x$ for an element in $L_{-1}$ with $f(a, x)=1, f(b, x)=0$. By Lemma 42 we have that $b x=0$ and also that $w=a x$ is a nonzero element of $H$ orthogonal to $v$. The lemma is proved if we show that $w$ is a scalar multiple of $v$. We shall derive a contradiction from the contrary assumption. By a harmless normalization we may assume that $w$ is of the form $u+\lambda v$. Then $a w-a$ is a scalar multiple of $b$. Now if we write $W$ for the one-dimensional subspace spanned by $b$, the hypotheses of Lemma 26 (a) are fulfilled (except that the element $u$ of that lemma is to be replaced by $w$ ). Hence

$$
b R_{a}^{p-2} R_{x}^{p-2}=b T_{1} T_{2} \cdots T_{p-2}
$$

where $T_{i}=i R_{w}+i_{2} I$. But the left side of (23) vanishes by Lemma 44 , while the right side is nonzero, since $i R_{w}+i_{2} I$ is nonsingular on $L_{1}$ for $1 \leqq i \leqq p-2$.

Lemma 46. If $R_{v}^{2} \neq 0$, then $f(u, u)=0$.

Proof. We may assume that $R_{0}^{2}$ is not zero on $L_{1}$. Pick $a$ in $L_{1}$ with $a R_{0}^{2} \neq 0$. Write $b=a v, c=b v$. The elements $a, b, c$ are linearly independent and thus we may pick elements $x, y, z$ in $L_{-1}$ dual to $a, b, c$ (relative to $f$ ). From Lemma 42 we have that $a z=0$, and that $b z$ is a nonzero element of $H$ orthogonal to $u$. We note that $R_{a}$ and $R_{z}$ commute. From this we first derive $b R_{a} R_{z}=b R_{z} R_{a}$ $\neq 0$, since $a$ is not annihilated by any nonzero element of $H$. Hence $b a \neq 0$. By Lemma $44, b R_{a}^{p-2}=0$.

The lemma will be proved if we show that $b z$ is a scalar multiple of $u$. Assume not; we may normalize so that $b z=v+\lambda u$. Let $i$ be the smallest integer such that $b R_{a}^{i}=0$ (necessarily $i \geqq 2$ ). Then

$$
0=b R_{a}^{i} R_{z}=b R_{z} R_{a}^{i}=(v+\lambda u) R_{a}^{i} .
$$

Now $u R_{a}^{i}=0$ and $v R_{a}=-b$. Hence $b R_{a}^{t-1}=0$, a contradiction.

LEMMA 47. $R_{0}^{3}=0$.

Proof. Assume that $R_{v}^{3}$ is not zero on $L_{1}$. Then we can find $a, b=a v$, $c=b v, d=c v$ linearly independent in $L_{1}$. Let $w, x, y, z$ be elements of $L_{-1}$ which are dual to $a, b, c, d$ relative to $f$. By Lemmas 42 and 46 we have $a x=c z=$ a nonzero multiple of $u$. We may normalize so that $a x=c z=u$. Further, we have $c x=a z=0$. By Lemma 35, $c R_{a}^{p-2} R_{x}^{p-2}=c T_{1} \cdots T_{p-2}$, and from this it follows as usual that $t=c R_{a}^{p-2}$ is not zero. Write $s=t c$. Then $f(s, c)=f(t c, c)=f(t, c c)=0$. Hence $f(s c, u)=f(s, c u)=f(s, c)=0$. It follows that $s c=t c \cdot c$ is a scalar multiple of $u$ (Lemma 46). Next we prove $t z=0$. This is done just as in $\S 3$. In brief: $c a \cdot z=-a, c R_{a}^{2} R_{z}=0, c R_{a}^{p-2} R_{z}=c R_{z} R_{a}^{p-2}$ $=0$. All the hypotheses of Lemma 19 are now fulfilled (with $b$ and $y$ replaced by $c$ and $z$ ). The conclusion $t=0$ is a contradiction. 
LEMMA 48. $R_{0}^{2}=0$.

Proof. Assume that $R_{0}^{2}$ does not vanish on $L_{1}$. Choose $a, b=a v, c=b v$ linearly independent in $L_{1}$, and $x, y, z$ dual to them (relative to $f$ ) in $L_{-1}$. Assembling the information in Lemmas 42 and 46 , we have $a x=b y=c z=\mathrm{a}$ nonzero multiple of $v$ which we can normalize to be $v ; a y=b z=$ a multiple of $u$; all other products between $a, b, c$ and $x, y, z$ vanish. We can prove $a y=u$. For let $a y=\lambda u$. Then from the Jacobi identity on $a, b, y$ we get $a b \cdot y=(1-\lambda) b$. But $f(a b \cdot y, y)=f(a b, y y)=0$, while $f(b, y)=1$. Hence $\lambda=1$.

Write ' for $R_{v}$ (an inner derivation). We note: $a^{\prime}=b, a^{\prime \prime}=c$. For any $r$ in $L$ we have $r^{\prime \prime \prime}=0$ by Lemma 47 . Then by Leibniz's formula

$$
0=(r a)^{\prime \prime \prime}=r^{\prime \prime \prime}+a^{\prime \prime \prime}+3 r^{\prime \prime} a^{\prime}+3 r^{\prime} a^{\prime \prime} \text {. }
$$

Hence

$$
r^{\prime \prime} b+r^{\prime} c=0 .
$$

Write $d=b a$, and note $d^{\prime}=c a, d^{\prime \prime}=c b$. Making use of the equations $a z=0$, $b z=u, c z=v$, we can systematically compute products with $z$, beginning with $d z=-a, d^{\prime} z=-b, d^{\prime \prime} z=0$. To (24), with $r=d$, apply $R_{z}$ on the right. The result is $d^{\prime \prime} \cdot b z+d^{\prime} z \cdot c+d^{\prime} \cdot c z=0, d^{\prime \prime} u-b c+d^{\prime} v=0,4 d^{\prime \prime}=0$ (note that $d$ and its derivatives are in $L_{2}$ ). Hence $d^{\prime \prime}=0$.

Next, let $e=d a$. We find $e^{\prime}=d^{\prime} a+d b, e^{\prime} z=d^{\prime} z \cdot a+d z \cdot b+d \cdot b z=-b a-a b$ $+d u=2 d$. Also $e^{\prime \prime}=2 d^{\prime} b+d c, e^{\prime \prime} z=2\left(d^{\prime} z \cdot b+d^{\prime} \cdot b z\right)+d z \cdot c+d \cdot c z=2 d^{\prime} u-a c$ $+d v=6 d^{\prime}$. To (24), with $r=e$, apply $R_{z}$ on the right. We obtain $0=e^{\prime \prime} z \cdot b$ $+e^{\prime \prime} u+e^{\prime} z \cdot c+e^{\prime} v=6 d^{\prime} b+3 e^{\prime \prime}+2 d c+e^{\prime \prime}$. Apply $R_{z}$ again: $0=6\left(d^{\prime} z \cdot b+d^{\prime} u\right)$ $+4 e^{\prime \prime} z+2(d z \cdot c+d v)=12 d^{\prime}+24 d^{\prime}-2 a c+2 d^{\prime}=40 d^{\prime}$. Hence $d^{\prime}=0, b=-d^{\prime} z$ $=0$, a contradiction. (This is the one place in the proof of Theorem 7 that characteristic 5 causes trouble.)

Lemma 49. $R_{v}$ acting on $L_{i}$ has at most one elementary divisor of degree two.

Proof. Assume the contrary for $i=1$. Then in $L_{1}$ we can find four linearly independent elements $a, b=a v, c, d=c v$; note that $b v=d v=0$. Let $w, x, y, z$ be elements of $L_{-1}$ dual to $a, b, c, d$ relative to $f$. By computations like that in Lemma 42, together with Lemma 45, we have $c x=c w=a y=a z=b w=d y=0$; $a w, b x, c y, d z$ are nonzero multiples of $v ; a x$ and $c z$ are nonzero elements orthogonal to $u$ (hence linearly independent of $v$ ). We can find a linear combination $r$ of $w$ and $x$ with $a r=u$, and similarly a linear combination $s$ of $y$ and $z$ with $c s=u$. We observe that $c r=a s=0$.

Next we note that $c d \cdot y=0$, directly from the Jacobi identity. Also $c d \cdot z=0$, for by the Jacobi identity it is a scalar multiple of $d$, which must vanish since it is orthogonal to $z$ (relative to $f$ ). Hence $c d \cdot s=0$.

By Lemma 35,

$$
c R_{a}^{p-3} R_{r}^{p-3}=c T_{1} T_{2} \cdots T_{p-3}
$$


where $T_{i}=i R_{u}+i_{2} I$. Hence $t=c R_{a}^{p-3}$ is not 0 . Again $c R_{a} R_{s}=c R_{s} R_{a}=-a$. Hence $t s=c R_{a}^{p-3} R_{s}=c R_{s} R_{a}^{p-3}=0$. This allows us to apply Lemma 35 again, as follows:

$$
t R_{c}^{4} R_{s}^{4}=t T_{1} T_{2} T_{3} T_{4}
$$

Since $t \in L_{-2}$, we see that the right side of (25) is not 0 . But $t R_{c}^{2} \in H, t R_{c}^{3}=\mathrm{a}$ linear combination of $c$ and $d, t R_{c}^{4}=$ a scalar multiple of $c d, t R_{c}^{4} R_{s}=0$. This contradiction completes the proof of Lemma 49.

Lemma 50. If $a \in L_{i}, x \in L_{-i}$, and av and $x v$ are not zero, then ax is not a scalar multiple of $v$.

Proof. If $a x$ is a scalar multiple of $v$, then by Lemma $45, f(a x, v)=0$, whence $f(a, x v)=0$. But by Lemma $49, x v$ spans $L_{-i} v$. Hence $f\left(a, L_{-i} v\right)=0$, $f\left(a v, L_{-i}\right)=0$, a contradiction since $a v$ is not zero.

We pause at this point to fix notation and collect information for the remainder of the proof of Theorem 7. Assume that $L_{1} v$ is not 0 . By Lemmas 48 and $49, L_{1}$ has a basis $a, b, c_{1}, \cdots, c_{r}$ with $a v=b, b v=c_{i} v=0$. Take a basis $x, y, z_{1}, \cdots, z_{r}$ for $L_{-1}$ which is dual relative to $f$. Since $R_{v}$ on $L_{-1}$ is the negative of the adjoint of $R_{v}$ on $L_{1}$, we have $y v=-x, x v=z_{i} v=0$. Computation like that in Lemma 42 reveals first that all products between $L_{1}$ and $L_{-1}$ vanish except: $a x, b y, a y, c_{1} z_{1}, \cdots, c_{r} z_{r}$. Next, $a x=b y=c_{i} z_{i}=$ a nonzero scalar multiple of $v$ which we can normalize to be $v$. There remains only the product $a y$. Suppose $a y=\lambda u+\alpha v$. From the Jacobi identity on $a, b, y$ we get $a b \cdot y$ $=(1-\lambda) b$. But $f(a b \cdot y, y)=f(a b, y y)=0$, while $f(b, y)=1$. Hence $\lambda=1$, $a y=u+\alpha v$. (We have here repeated a fragment of the proof of Lemma 48.)

Set $w=y-\alpha x$; then $a w=u$. The element $w$ is convenient for our purposes since the triple $a, w, u$ fits the hypotheses of Lemmas 35-37 (with $w$ replacing $x)$.

Lemma 51. $\left(L_{i} v \cdot L_{i}\right) L_{-i}=0$.

Proof. We take $i=1$, and use the notation fixed above. Since $L_{1} v$ is spanned by $b$, what we have to show is that $a b$ and $b c_{i}$ annihilate $L_{-1}$. Everything comes right out of the Jacobi identity except the fact that $a b \cdot y=0$, which was noted just above.

Lemma 52. For $2 \leqq j \leqq(p-1) / 2, R_{a}$ maps $L_{j}$ one-to-one into $L_{j+1}$, and $R_{w}$ maps $L_{j+1}$ onto $L_{j}$. For $(p+1) / 2 \leqq j \leqq p-2, R_{w}$ maps $L_{j}$ one-to-one into $L_{j-1}$, and $R_{a}$ maps $L_{j-1}$ onto $L_{j}$.

Proof. This is immediate from Lemma 37 as soon as we check $L_{0} R_{a}^{2} R_{w}$ $=0, L_{0} R_{w}^{2} R_{a}=0$. But both statements follow from Lemma 51 .

Lemma 53. $R_{r}$ annihilates either $L_{i}^{2}$ or $L_{-i}^{2}$.

Proof. Assume the contrary for $i=1$, and use the notation above. $L_{1}^{2}$ is 
spanned by $a b, a c_{j}, b c_{j}(j=1, \cdots, r)$. The elements $a b, b c_{j}$ are visibly annihilated by $v$. Hence some $a c_{j} \cdot v \neq 0$. Similarly some $y z_{k} \cdot v \neq 0$. It then follows from Lemma 50 (with $i=2$ ) that $a c_{j} \cdot y z_{k}$ is not a multiple of $v$. But we are able to compute this product and find that it is a multiple of $v$. We have $c_{j} y=0, a z_{k}=0, a y=u+\alpha v, b y=v, c_{j} z_{k}=0$ or $v$. By repeated use of the Jacobi identity $a c_{j} \cdot y=-c_{j}, a c_{j} \cdot z_{k}$ is a multiple of $b, a c_{j} \cdot y z_{k}$ is a linear combination of $c_{j} z_{k}$ and $b y$ and is a multiple of $v$.

\section{Lemma 54. $R_{v}$ annihilates $L_{i}^{2}$.}

Proof. Assume the contrary for $i=1$. Continuing the argument of Lemma 53 , we have that some $a c_{j}$ is not annihilated by $R_{v}$, that is, $b c_{j} \neq 0$. Let us write simply $c$ for $c_{j}$.

By Lemma 35, $c R_{a}^{i} R_{w}=c T_{i} R_{a}^{i-1}$. Since $c \in L_{1}$ and $T_{i}=i R_{u}+i_{2} I, c T_{i}$ is simply $(i+1)_{2} c$. Thus

$$
c R_{a}^{i} R_{w}=(i+1)_{2} c R_{a}^{i-1} .
$$

We apply (26) with $i=p-2$. By Lemma $53, L_{-1}^{2} v=0$ (since we are assuming $\left.L_{1}^{2} v \neq 0\right)$. Now $c R_{a}^{p-2} R_{w} \in L_{-1}^{2}$. Hence $c R_{a}^{p-3} R_{v}=0$. We are going to contradict this by a computation which is a slight variant of numerous earlier ones.

We have $R_{a} R_{x}=R_{x} R_{a}+R_{v}$. Since $c v=c x=0$, we deduce $c R_{a} R_{x}=0$ and further

$$
c R_{a}^{2} R_{x}=c R_{a} R_{v}=c b
$$

We next note

$$
c R_{a}^{2} R_{v} R_{w}=4 c b
$$

To prove (28) we write $R_{v} R_{w}=R_{w} R_{v}+R_{x}$ (recall that $w=y-\alpha x$ so that $w v=y v=-x)$, use (27), and (26) with $i=2$.

We are going to establish inductively

$$
c R_{a}^{i} R_{v} R_{w}^{i-1}=(i+2)(i+1)_{2} i_{2} \cdots 5_{2} 4_{2} c b
$$

for $i \geqq 2$. For $i=2$ we interpret (29) to coincide with (28); at any rate the reader can check that the induction can start correctly this way. In (29) replace $R_{v} R_{w}$ by $R_{w} R_{v}+R_{x}$. The first of the resulting terms is

$$
(i+1)_{2}(i+1) i_{2}(i-1)_{2} \cdots 4_{2} c b
$$

by (26) and induction. We study the remaining term $c R_{a}^{1} R_{x} R_{w}^{1-2}$. By Lemma 51, $x w$ annihilates $L_{1}$; in particular $x w$ annihilates $a, c$ and all their products. It follows that $R_{x}$ and $R_{w}$ may be interchanged in the expression. The $R_{w}$ thus made adjacent to $c R_{a}^{i}$ can be absorbed as in (26). Then we are ready to commute $R_{w}$ and $R_{x}$ again. By a succession of these steps we eventually push $R_{x}$ all the way to the right, arriving at 


$$
(i+1)_{2} i_{2} \cdots 4_{2} c R_{a}^{2} R_{x}=(i+1)_{2} \cdots 4_{2} c b
$$

by (27). Since (30) and (31) add up to the right side of (29), the induction is complete.

Set $i=p-3$ in (29). We saw that the left side vanishes. The coefficient on the right is nonzero, and further $c b$ is nonzero by assumption. This contradiction concludes the proof of Lemma 54.

Lemma 55. $L_{2 i} v \cdot L_{-i}=0$.

Proof. It suffices to prove $f\left(L_{2 i} v \cdot L_{-i}, L_{-i}\right)=0$, or $f\left(L_{2 i} v, L_{-i}^{2}\right)=0$, or $f\left(L_{2 i}, v L_{-i}^{2}\right)=0$, and this is true by Lemma 54 .

We record for later use three more computational lemmas. For conciseness we introduce a new symbol: $F_{i}=i(i+3) / 2$.

LEMMA 56. For $i \geqq 1, b R_{a}^{\imath} R_{w}=F_{i-1} b R_{a}^{i-1}$.

Proof. By Lemma $51, b a \cdot w=0$. We can then apply Lemma 35 to deduce (ba) $R_{a}^{i-1} R_{w}=b a T_{i-1} R_{a}^{i-1}$. Here $T_{i-1}=(i-1) R_{u}+(i-1)_{2} I$. Since $(a b) R_{u}=2 a b$, we compute that $(a b) T_{i-1}=F_{i-1} a b$.

LEMma 57. For $i \geqq 2$

$$
b R_{a}^{i} R_{x} R_{w}^{i-2}=0 .
$$

Proof. For $i=2$ we have to prove $b R_{a}^{2} R_{x}=0$, and this admits direct verification. We assume (32) known with $i$ replaced by $i-1$. In (32) replace $R_{x} R_{w}$ by $R_{w} R_{x}+R_{x w}$. By Lemma 51 (since $w v=-x$ ), $x w$ annihilates $a$ and $b$ and hence all their products. This term therefore drops out. To the remaining term apply Lemma 56 and our inductive assumption.

LEMMA 58. For $i \geqq 1, b R_{a}^{i} R_{v} R_{w}^{i-1}=0$.

Proof. The case $i=1\left(b R_{a} R_{v}=0\right)$ is immediate. Replace $R_{v} R_{w}$ by $R_{w} R_{v}+R_{x}$. The first resulting term vanishes by Lemma 56 and induction. The second vanishes by Lemma 57 .

Lemma 59. Suppose that $R_{v}$ vanishes on every $L_{i}$ except for $i= \pm 1$. Let $S$ denote the subspace of $L$ spanned by $v, b, b R_{a}, \cdots, b R_{a}^{p-3}, x, x R_{w}, \cdots, x R_{w}^{p-3}$. Then $S$ is an ideal in $L$.

Proof. We begin by noting that $S$ is invariant under $R_{a}$ and $R_{w}$; by symmetry it suffices to handle $R_{w}$. We have $w v=-x, b w=v$. By Lemma 44, $x R_{w}^{p-3}$ is annihilated by $R_{w}$. Lemma 56 covers the application of $R_{w}$ to $b R_{\underline{a}}^{4}$.

Let $T$ denote the set of elements $t$ satisfying $t L \subset S$. $T$ is invariant under $R_{a}$ and $R_{w}$ : for instance $t w \cdot L \subset t L \cdot w+t \cdot w L \subset S w+S \subset S$. Since $L_{1} v$ and $L_{-1} v$ are spanned by $b$ and $x$, and all other $L_{i} v$ are 0 , we have $v \in T$. It follows that $v R_{a}^{i}$ and $v R_{w}^{i}$ lie in $T$ for all $i$. This proves $S \subset T$, and $S$ is an ideal in $L$.

$\mathrm{Up}$ to this point we have in effect been analyzing the structure of any 
restricted Lie algebra of rank two with a nonsingular invariant form; simplicity has not yet been invoked. Possibly the full structure of this class of algebras can be elucidated (it is a fact that examples do exist); but we shall make use of the simplicity from now on.

In the next four lemmas we maintain our assumption that $L_{1} v$ is nonzero and continue to use the notation we have established.

LemMA 60. $a b$ and $x y$ are not 0.

Proof. We shall assume $a b=0$ and derive a contradiction by showing that $L_{j} v=0$ for $j \neq \pm 1$ (this, by Lemma 59 , provides a proper ideal in $L$ ).

First we prove $L_{j} b=0$ for $2 \leqq j \leqq(p-1) / 2$. This is true for $j=(p-1) / 2$ by Lemma 55 (take $i=1 / 2$ ). We make a descending induction on $j$. We suppose $L_{j+1} b=0$ known $(2 \leqq j<(p-1) / 2)$. Then $L_{j} R_{a} R_{b}=0$. But $R_{a}$ and $R_{b}$ commute; hence $L_{j} R_{b} R_{a}=0$. On $L_{j+1}, R_{a}$ is one-to-one (Lemma 52); hence $L_{j} b=0$.

We already know $b c_{1}=\cdots=b c_{r}=0$ by Lemma 54 . The hypothesis $b a=0$ tells us therefore that $L_{1} b$ is 0 , whence $L_{2} R_{w} R_{b}=0$. So for any $j$ with $2 \leqq j$ $\leqq(p-1) / 2, L_{j} v=L_{j} \cdot b w \subset L_{j}\left(R_{b} R_{w}+R_{w} R_{b}\right)=0$, as desired.

LEMMA 61. $L_{2}$ contains an element d satisfying $d y=a$.

Proof. Since $x y \neq 0$ (Lemma 58) there exists an element $d \in L_{2}$ with $f(d, \dot{x} y) \neq 0$. Now $d y$ lies in $L_{1}$ and is orthogonal to $y$, relative to $f$. Hence $d y$ is a linear combination of $a, c_{1}, \cdots, c_{r}$. The $a$-component must actually be present, for otherwise we have the contradiction $f(d y, x)=0$. Since $a c_{i} \cdot y$ $=-c_{i}$ by the Jacobi identity, we can adjust $d$ by a suitable linear combination of the elements $a c_{i}$ so as to have $d y=a$ (after a further normalization by a scalar).

LEMḾA 62. $\operatorname{Dim}\left(L_{2}\right) \geqq \operatorname{dim}\left(L_{1}\right)$.

Proof. We prove this by showing that the elements $a b, a c_{1}, \cdots, a c_{r}$ and the element $d$ of Lemma 61 are linearly independent. Under $R_{y}, a c_{i}$ is sent into $-c_{i}, d$ into $a$, and $a b$ into 0 (Lemma 51). Together with the fact that $a b$ is nonzero, this proves the linear independence.

Lemma 63. $\operatorname{Dim}\left(L_{(p-1) / 2}\right) \geqq \operatorname{dim} L_{1}$. If the two dimensions are equal, then all root spaces $L_{j}(j \neq 0)$ have the same dimension.

Proof. In the sequence of spaces $L_{1}, L_{2}, \cdots, L_{(p-1) / 2}$ the dimensions are increasing (in the weak sense); the step from $L_{1}$ to $L_{2}$ is covered by Lemma 62 , snd the remaining steps by Lemma 52. This implies both statements in the present lemma.

LEMMA 64. If for some $i$ the root spaces $L_{i}$ and $L_{2 i}$ are both not annihilated by $R_{v}$, then all root spaces $L_{j}(j \neq 0)$ have the same dimension.

Proof. We have $\operatorname{dim}\left(L_{2 i}\right) \geqq \operatorname{dim}\left(L_{i}\right)$ by Lemma 62 . Now let $L_{2 i}$ play the 
role of $L_{1}$ in Lemma $63 ; L_{i}$ becomes $L_{(p+1) / 2}$ and its dimension is the same as that of $L_{(p-1) / 2}$. The hypothesis of Lemma 63 is therefore fulfilled.

It is our intention to prove that in all cases the root spaces have equal dimension. The discussion need only continue under the assumption $L_{1} v \neq 0$, $L_{2} v=0$. The crucial fact then is that the element $d$ of Lemma 61 satisfies $d v=0$. Applying $R_{v}$ to the equation $d y=a$ we then compute

$$
d x=-b .
$$

Recalling that $w=y-\alpha x$, we find from (33):

$$
d w=a+\alpha b .
$$

Apply the Jacobi identity to the triple $d, x, w$. The result is:

$$
d \cdot x w=-2 v .
$$

We proceed to more elaborate computations of products involving $d$.

LEMma 65. For $i \geqq 1$,

$$
d R_{a}^{i} R_{w}=F_{i} d R_{a}^{i-1}+\alpha b R_{a}^{i}
$$

where, as in Lemma 56, $F_{i}=i(i+3) / 2$.

Proof. We check this first for $i=1$, using $R_{a} R_{w}=R_{w} R_{a}+R_{u}, d u=2 d$, and (34). Assume (36) for $i-1$. Then

$$
\begin{aligned}
d R_{a}^{i} R_{w} & =d R_{a}^{i-1}\left(R_{w} R_{a}+R_{u}\right) \\
& =\left(F_{i-1} d R_{a}^{i-2}+\alpha b R_{a}^{i-1}\right) R_{a}+(i+1) d R_{a}^{i-1},
\end{aligned}
$$

and this boils down to the right side of (36).

Lemma 66. For $i \geqq 1$,

$$
d R_{a}^{i} R_{x} R_{w}^{i-1}=G_{i} b a
$$

where $G_{1}=-1, G_{2}=-3$,

$$
G_{i}=F_{i} G_{i-1}+2 F_{i-2} \cdots F_{2} F_{1} .
$$

Proof. We have $R_{a} R_{x}=R_{x} R_{a}+R_{v}$. Then $d R_{a} R_{x}=d x \cdot a+d v=-b a$ by (33). This checks $(37)$ for $i=1$. We begin the induction at this point, making a special remark on $i=2$ at the appropriate moment. In (37) replace $R_{x} R_{w}$ by $R_{w} R_{x}+R_{x w}$. On the first resulting term we use Lemma 65 , obtaining

$$
\left(F_{i} d R_{a}^{i-1}+\alpha b R_{a}^{i}\right) R_{x} R_{w}^{i-2} \text {. }
$$

The second of the terms in (39) vanishes by Lemma 57. The first, by induction, is $F_{i} G_{i-1} b a$. It remains to discuss the term $d R_{a}^{i} R_{x w} R_{w}^{i-2}$. Since $x w$ annihilates $a$ (Lemma 51) we can commute $R_{x w}$ past $R_{a}^{t}$. Quoting (35), we reach 
$-2 v R_{a}^{i} R_{w}^{i-2}=2 b R_{a}^{i-1} R_{w}^{i-2}$. By using Lemma 56 repeatedly we identify this as $2 F_{i-2} \cdots F_{2} F_{1} b a$ (for $i=2$ it is simply $2 b a$, and this leads to the value $G_{2}$ $=-3$, since $F_{2} G_{1}+2=5(-1)+2=-3$ ). Summarizing: the left side of (38) equals $F_{i} G_{i-1} b a+2 F_{i-2} \cdots F_{2} F_{1} b a$ as was to be shown.

Lemma 67. For $i \geqq 0$

$$
d R_{a}^{i} R_{v} R_{w}^{i}=H_{i} b a
$$

where $H_{0}=0$,

$$
H_{i}=F_{i} H_{i-1}+G_{i} .
$$

Proof. For $i=0$, the left side of (40) is $d v=0$, and the right side is 0 too. Then use $R_{v} R_{w}=R_{w} R_{v}+R_{x}$, which follows from $w v=-x$.

$$
\begin{aligned}
d R_{a}^{i} R_{v} R_{w}^{i} & =d R_{a}^{i}\left(R_{w} R_{v}+R_{x}\right) R_{w}^{i-1} \\
& =\left(F_{i} d R_{a}^{i-1}+\alpha b R_{a}^{i}\right) R_{v} R_{w}^{i-1}+d R_{a}^{i} R_{x} R_{w}^{i-1} \\
& =F_{i} H_{i-1} b a+G_{i} b a
\end{aligned}
$$

by Lemma 65 , induction, Lemma 58 , and Lemma 66 .

Let us compute the values of $F_{i}, G_{i}, H_{i}$ up to $i=4 . F_{1}=2, F_{2}=5, F_{3}=9, F_{4}$ $=14$. Now use (38). $G_{3}=9(-3)+4=-23, G_{4}=14(-23)+2 \cdot 5 \cdot 2=-302$. Next use (41). $H_{1}=-1, H_{2}=5(-1)-3=-8, H_{3}=9(-8)-23=-95, H_{4}$ $=14(-95)-302=-1632$.

Since $H_{1}$ is not 0 , we have, by (40), that $d R_{a} R_{v} \neq 0$. Now $d a \in L_{3}$; hence $L_{3} v \neq 0$. If $H_{4}$ is not 0 , we similarly have $d R_{a}^{4} R_{v} \neq 0$ and $L_{6} v \neq 0$. Then Lemma 64 tells us that all root spaces have the same dimension. Now $H_{4}=-1632$ is divisible by 2,3 and 17 . So there is trouble for characteristic 17 . But the trouble is easily resolved by a slight additional argument. We have in any event $L_{3} v \neq 0$. If $L_{6} v \neq 0$, all is well. If $L_{6} v=0$, we can replace $L_{1}$ by $L_{3}$ in the argument we have just carried out. Conclusion: $L_{9} v \neq 0$. But $1=2.9$ for characteristic 17 , so Lemma 64 is applicable again. We have proved:

Lemma 68. All root spaces $L_{j}(j \neq 0)$ have the same dimension.

This enables us to sharpen Lemma 52.

Lemma 69. For $2 \leqq j \leqq p-3, R_{a}$ induces a one-to-one linear transformation of $L_{j}$ onto $L_{j+1}$ and $R_{w}$ induces a one-to-one linear transformation of $L_{j+1}$ onto $L_{j}$.

Lemma 70. Assume $L_{2} v \neq 0$. Then $L_{2} v$ is spanned by $a b ; L_{-2} v$ is spanned by $x y$.

Proof. By symmetry it suffices to establish the second statement. Since $L_{-2} v$ is one-dimensional (Lemma 49) and $x y$ is nonzero (Lemma 60), it suffices to prove $x y \in L_{-2} v$. It is equivalent to prove $f(x y, N)=0$ where $N$ is the null space of $R_{v}$ within $L_{2}$. 
As we saw in Lemma 62 , the elements $d, a b$, and $a c_{i}(1 \leqq i \leqq r)$ span a subspace of $L_{2}$ with the same dimension as that of $L_{1}$. Now that we know that $L_{1}$ and $L_{2}$ have the same dimension, we have a basis of $L_{2}$ consisting of these elements. Since $R_{v}$ annihilates $a b$ and $a c_{i}$ (Lemma 54), and since $N$ has codimension one in $L_{2}, a b$ and $a c_{i}$ constitute a basis of $N$. We know that $x$ annihilates $a b$ and $a c_{i}$. Hence $f(x y, N)=0$.

From Lemma 70 we are able to deduce that (33), previously established only on the assumption that $L_{2} v$ is 0 , also holds when $L_{2} v$ is nonzero. For $d v$ is a scalar multiple of $a b$ and $y \cdot a b=0$. Applying $R_{v}$ to the equation $d y=a$ then yields (33); $d x=-b$. Equation (34) and (35) follow as before.

Lemma 71. The null space of $R_{w}$ within $L_{2}$ is spanned by $a b$.

Proof. We examine $R_{w}$ on the basis $d, a b, a c_{i}$ of $L_{2}$. We have $d w=a+\alpha b$, $a c_{i} \cdot w=-c_{i}\left(\right.$ recall $\left.c_{i} w=0, a w=u\right)$. Since $a b \cdot w=0$, and since the elements $a+\alpha b, c_{1}, \cdots, c_{r}$ are linearly independent, the lemma follows.

Lemma 72. For $3 \leqq j \leqq p-2, L_{j} R_{x w}$ is spanned by $b R_{a}^{j-3}$.

Proof. First we examine $R_{x w}$ on $L_{2}$. Since $x w$ annihilates $a b$ and $a c_{i}$, and since $d \cdot x w=-2 v$ by (35), $L_{2} R_{x w}$ is spanned by $v$. For $3 \leqq j \leqq p-2$ we have $L_{j}=L_{2} R_{a}^{j-2}$ by Lemma $69 . R_{a}$ and $R_{x w}$ commute; consequently $L_{j} R_{x w}$ $=L_{2} R_{x w} R_{a}^{j-2}$, which is spanned by $v R_{a}^{j-2}=-b R_{a}^{j-3}$.

LEMMA 73. For $2 \leqq j \leqq p-1, L_{j} x$ is spanned by $b R_{a}^{j-2}$.

Proof. (a) $j=2$. We must examine the products $d x, a b \cdot x, a c_{i} \cdot x$, and we find $d x=-b, a b \cdot x=a c_{i} x=0$.

(b) $2<j<p-1$. Since $R_{w}$ is one-to-one on $L_{j}$ (Lemma 69), and $b R_{a}^{j-2} R_{w}$ is a scalar multiple of $b R_{a}^{j-3}$ (Lemma 56), it suffices to prove that $L_{j} R_{x} R_{w}$ is spanned by $b R_{a}^{j-3}$. Now

$$
L_{j} R_{x} R_{w} \subset L_{j} R_{w} R_{x}+L_{j} R_{x w} .
$$

The first term on the right of (42) is contained in $L_{j-1} R_{x}$ which is spanned by $b R_{a}^{j-3}$ by induction. The second term is covered by Lemma 72 .

(c) A special argument is needed for $x L_{p-1}$. We know that $x L_{-1}$ is spanned by $x w$, and so our problem is to prove that $x w$ is a scalar multiple of $b R_{a}^{p-3}$. By Lemma 44, $x w$ is annihilated by $R_{w}^{p-3}$. Thus $x R_{w}^{p-3}$ is an element of $L_{2}$ annihilated by $R_{w}$. By Lemma $71, x R_{w}^{p-3}$ is a scalar multiple of $a b$. But by iterated use of Lemma $56, b R_{a}^{p-3} R_{w}^{p-4}$ is a nonzero scalar multiple of $a b$. So: we have two elements, namely $x w$ and $b R_{a}^{p-3}$ in $L_{-2}$; on applying $R_{w}^{p-4}$ they both become scalar multiples of $a b$. By Lemma $69, R_{w}^{p-4}$ is a one-to-one map of $L_{-2}$ into $L_{2}$. Since $b R_{a}^{p-3}$ is nonzero, we conclude that $x w$ is a scalar multiple of $b R_{a}^{p-3}$.

The moment has arrived for concluding the proof of Theorem 7 : we shall produce a proper ideal in $L$. It is the subspace $S$ spanned by $x, v, b, b R_{a}, b R_{a}^{2}, \cdots$, 
$b R_{a}^{p-3}$. Note that this sequence is obtained from $x$ by repeated operation of $R_{a}$, and that a final application of $R_{a}$ yields 0 by Lemma 44. Hence $S a \subset S$. As in the proof of Lemma 59, all will be done as soon as we verify $x L \subset S$. The action of $R_{x}$ on the spaces $L_{2}, \cdots, L_{p-1}$ is covered by Lemma 73. On the two remaining spaces $L_{0}$ and $L_{1}$ we have known all along that $L_{0} x$ is spanned by $x$ and $L_{1} x$ is spanned by $v$. The proof is complete.

\section{Part III. Appendix on characteristics two and three}

12. Rank one, characteristic two. The counterpart of Theorem 2 for the case of characteristic two is very easy-see Lemma 74 . However we can go further and analyze any collection of roots.

THEOREM 8. Let $L$ be a simple Lie algebra of rank one over an algebraically closed field of characteristic two. Let $\left\{L_{\alpha}\right\}$ denote the root spaces relative to a one-dimensional Cartan subalgebra. Then each $L_{\alpha}(\alpha \neq 0)$ is two-dimensional, and the roots form a group under addition.

As usual we write $u$ for a nonzero element of the Cartan subalgebra. The product of any two elements of $L_{\alpha}$ is a scalar multiple of $u$. Suppose $L_{\alpha}^{2} \neq 0$. Then we can find $x, y \in L_{\alpha}$ with $x y=u$ (note that $x$ and $y$ are necessarily linearly independent). Let $z$ be a possible third linear independent element in $L_{\alpha}$. Apply the Jacobi identity to the triple $x, y, z$. The result: $z u$ is a linear combination of $x u$ and $y u$. Since $R_{u}$ is nonsingular on $L_{\alpha}$, this is impossible. Hence:

Lemma 74. For any root space $L_{\alpha}$, either $L_{\alpha}^{2}=0$ or $L_{\alpha}$ is two-dimensional.

From this, it takes just a moment to dispose of the restricted case: there are no simple restricted Lie algebras of rank one and characteristic two.

Lemma 75. If $L_{\alpha}^{2} \neq 0$ and $x$ is an element of $L$ annihilating $L_{\alpha}$, then $x=0$.

Proof. There exist elements $a, b$ in $L_{\alpha}$ with $a b=u$. Applying the Jacobi identity to $a, b, x$ we find $u x=0$. Hence $x$ is a scalar multiple of $u$. But $R_{u}$ is nonsingular on $L_{\alpha}$. Hence $x=0$.

Lemma 76. Suppose $\alpha, \beta, \gamma$ are roots with $\beta+\gamma=\alpha, L_{\alpha}^{2} \neq 0, L_{\beta}^{2}=L_{\gamma}^{2}=0$. Then $L_{\beta} L_{\gamma}=0$.

Proof. Let $y \in L_{\beta}, z \in L_{\gamma}, y z=x$. We have $x L_{\alpha}=y z \cdot L_{\alpha} \subset y L_{\alpha} \cdot z+z L_{\alpha} \cdot y \subset L_{\gamma}^{2}$ $+L_{\beta}^{2}=0$. By Lemma 75, $x=0$.

Lemma 77. Let $\alpha$ be a root such that $L_{\alpha}^{2} \neq 0$. Let $\beta$ be any other root, and $\gamma=\alpha+\beta$. Then $\gamma$ is also a root. If $L_{\beta}$ is two-dimensional, so is $L_{\gamma}$.

Proof. If $\gamma$ is not a root then $L_{\alpha} L_{\beta}=0$, which is impossible by Lemma 75 .

Suppose $L_{\beta}$ is two-dimensional. Let $x, y$ be elements of $L_{\alpha}$ with $x y=u$. We have $R_{x} R_{y}+R_{y} R_{x}=R_{u}$. The subspace $L_{\beta}+L_{\gamma}$ is invariant under $R_{x}$ and 
$R_{y}$; hence the trace of $R_{u}$ on $L_{\beta}+L_{\gamma}$ is 0. On $L_{\beta}$, the trace of $R_{u}$ is 0 . On $L_{\gamma}$, the trace of $R_{u}$ is $\gamma$ multiplied by the dimension of $L_{\gamma}$. Hence that dimension must be even.

Because of the equation $R_{x} R_{y}+R_{y} R_{x}=R_{u}$, the null spaces of $R_{x}$ and $R_{y}$ on $L_{\gamma}$ have zero intersection. If the dimension of $L_{\gamma}$ is $k$, these null spaces have dimension at least $k-2$ (since the range is in $L_{\beta}$, which is two-dimensional). Hence $k$ must be 2 or 4 . It remains for us to exclude the possibility $k=4$.

We again pick elements $x, y$ in $L_{\alpha}$ with $x y=u$, this time in Jordan form: $x u=\alpha x, y u=\alpha y+\lambda x, \lambda=0$ or 1 . Let $N$ denote the (necessarily two-dimensional) null space of $R_{x}$ in $L_{\gamma}$. From the equation $\left[R_{x} R_{u}\right]=\alpha R_{x}$ we see that $N$ is invariant under $R_{u}$. Since $R_{y} R_{x}=R_{u}$ on $N$ we have that $R_{x}$ is a one-to-one mapping of $L_{\beta}$ onto $N$. Pick a nonzero element $a$ in $N$ with $a u=\gamma a$. Write $a y=c, c y=e$. We apply the Jacobi identity five times in succession, listing the triple and the resulting equation. (a) $a, x, y: c x=\gamma a$. (b) $a, y, u: c u=\beta c$. (c) $c, x, y: e x=\alpha c$. (d) $c, y, u: e u=\gamma c+\lambda \gamma a$. (e) $e, x, y: e y \cdot x=\beta e+\lambda \gamma a$. Since $e y \in L_{\beta}$ and $R_{x}$ maps $L_{\beta}$ into $N$, we deduce $e \in N$. But this means $e x=0$, whereas $e x=\alpha c \neq 0$.

With these preliminary lemmas at our disposal, we are ready to give the proof of Theorem 8. Write $\Gamma$ for the set of all roots $\gamma$ such that $L_{\gamma}^{2} \neq 0$. Let $\Delta$ be the group generated by $\Gamma$ (i.e. the set of all sums of elements in $\Gamma$ ). We have three things to prove: (1) Every member of $\Delta$ is a root, (2) For $\delta \in \Delta$, $L_{\delta}$ is two-dimensional, (3) Every root lies in $\Delta$.

(1) Let $\delta \in \Delta, \delta=\gamma_{1}+\gamma_{2}+\cdots+\gamma_{n}, \gamma_{i} \in \Gamma$. We may assume by induction on $n$ that $\zeta=\gamma_{2}+\cdots+\gamma_{n}$ is a root. By Lemma $77, \delta=\gamma_{1}+\zeta$ is a root.

(2) We may further assume by induction that $L_{\zeta}$ is two-dimensional. By the second half of Lemma 77, $L_{\delta}$ is also two-dimensional.

(3) (This is the sole portion of the proof that uses simplicity.) Let $S$ denote the subspace of $L$ spanned by $\left\{L_{\alpha}\right\}$, where $\alpha$ is not in $\Delta$. We shall prove that $S$ is an ideal. Given a root $\alpha$ not in $\Delta$, and any root $\beta$, we must prove $L_{\alpha} L_{\beta} \subset S$. If $\alpha+\beta$ is not in $\Delta$, all is well. Assume $\alpha+\beta=\gamma_{1}+\gamma_{2}+\cdots$ $+\gamma_{n}, \gamma_{i} \in \Gamma$. We shall in this case prove $L_{\alpha} L_{\beta}=0$. This is immediate from Lemma 76 if $n=1$ (note that $L_{\alpha}^{2}=L_{\beta}^{2}=0$ since $\alpha$ and $\beta$ are not in $\Gamma$; they are not even in $\Delta$ ). We make an induction on $n$. The roots $\alpha+\gamma_{1}$ and $\beta$ are not in $\Delta$ and they add up to a root in $\Delta$. By our inductive assumption $L_{\alpha+\gamma_{1}} L_{\beta}=0$. Similarly $L_{\alpha} L_{\beta+\gamma_{1}}=0$. By the Jacobi identity, $\left(L_{\alpha} L_{\beta}\right) L_{\gamma_{1}}=0$, whence, by Lemma $75, L_{\alpha} L_{\beta}=0$. This completes the proof of Theorem 8 .

To conclude this section we give an example showing that there is a genuine distinction between the sets $\Gamma$ and $\Delta$ introduced above, i.e. that in a simple Lie algebra there may exist a root space $L_{\alpha}$ with $L_{\alpha}^{2}=0$. The smallest possible dimension for an example is 7 . In the following example the Cartan subalgebra is spanned by $u$; there are three root spaces $L_{\alpha}, L_{\beta}, L_{\gamma}$ $(\gamma=\alpha+\beta)$ spanned by $a, b \in L_{\alpha}, p, q \in L_{\beta}, r, s \in L_{\gamma} . R_{u}$ acts diagonally on its root spaces. We have $p q=u, r s=u$, but $a b=0$ so that $L_{\alpha}^{2}=0$. The remaining 
products are as follows: $a p=0, a q=s, a r=p, a s=0, b p=\beta s, b q=r, b r=\beta q$, $b s=p, p r=\gamma b, p s=\alpha a, q r=0, q s=b$. Brutal computation verifies the Jacobi identity and the simplicity.

13. Rank one, characteristic three. We are able to establish the analogue of Theorem 2 by finding the simple algebras whose only roots are \pm 1 .

Theorem 9. Let $L$ be a simple Lie algebra over an algebraically closed field of characteristic three. Assume that a one-dimensional Cartan subalgebra of $L$ is spanned by an element $u$ such that the characteristic roots of $R_{u}$ are $1,-1,0$. Then $L$ is either 3-dimensional or isomorphic to a certain 7-dimensional algebra.

Proof. Write $V$ and $W$ for the root spaces $L_{1}$ and $L_{-1}$ respectively. We begin by recalling Lemma 5 : if $a \in V$ and $W a \neq 0$ then the only elements of $V$ annihilated by $a$ are the scalar multiples of $a$.

If $V^{3}=W^{3}=0$, it is readily seen that $V^{2}+W^{2}$ is an ideal in $L$, hence 0 . This makes $V$ and $W$ one-dimensional, $L$ 3-dimensional. We therefore assume $V^{3} \neq 0$ and select elements $a, b, c \in V$ with $a \cdot b c=u$. Write $b c=x, c a=y$, $a b=\boldsymbol{z}$.

If $r$ and $s$ are any elements of $V$, we claim $r \cdot r s=0$. To see this, note that $r \cdot r s$ and $s \cdot r s$ are scalar multiples of $u$, say $\alpha u$ and $\beta u$. From the Jacobi identity on $r, s, r s$ we get $\beta u r-\alpha u s=0$. If $r$ and $s$ are linearly dependent, there is nothing to prove. Otherwise there is a contradiction unless $\alpha=\beta=0$.

From this we get the following vanishing products: $a y=a z=b x=b z=c x$ $=c y=0$.

Apply the Jacobi identity to the triples $y, a, b$ and $c, a, z$. The result is

$$
y z=-a \cdot b y=-a \cdot c z .
$$

It follows from (43) that $b y$ and $c z$ are equal, say to $\lambda u$. The Jacobi identity on $a, b, c$ gives us $a x+b y+c z=0$. Since $a x=u$, we find $1+2 \lambda=0, \lambda=1$, $b y=c z=u$. Since $y z$ annihilates $a$, it is a scalar multiple of $a$. From (43) we deduce simultaneously $y z=-a, a u=a$. By symmetry $(a, b$ and $c$ are now on an equal footing), $z x=-b, x y=-c, b u=b, c u=c$. The Jacobi identity on $u, b, c$ yields $x u=-x$ and similarly $y u=-y, z u=-z$. We have identified all products in the 7-dimensional algebra spanned by $u, a, b, c, x, y, z$ and recognize it, for instance, as the Cayley numbers of trace 0 under commutation. Finally we must show that $L$ contains nothing else. Think of the equation $R_{b} R_{c}-R_{c} R_{b}=R_{x}$ applied to $W$. If the dimension of $W$ is $n$, the range of $R_{x}$ on $W$ is $(n-1)$-dimensional (since $R_{x}$ annihilates only $x$ ). But the range of $R_{b} R_{c}-R_{c} R_{b}$ is spanned by $b$ and $c$. Hence $n$ is 3 . This proves that $V$ and $W$ are 3-dimensional and completes the proof of Theorem 9.

14. Rank two, nondiagonable case. In the case of a simple restricted Lie algebra of rank two and characteristic 2 or 3 , we can rule out the nondiagonable case without the aid of an invariant form. Moreover, the proof is much simpler than that of Theorem 7. 
THEOREM 10. Let $L$ be a simple restricted Lie algebra of rank two over an algebraically closed field of characteristic 2 or 3. Then any two-dimensional Cartan subalgebra $H$ acts diagonally.

The first step in the proof can be taken for any $p$. We have a basis $u, v$ for $H$ with $u^{p}=u, v^{p}=0$. Write ' for $R_{v}$, and $U$ for the one-dimensional subspace spanned by $u$.

Lemma 78. If $a \in L_{1}, a^{\prime} R_{a}^{p-2} \neq 0$, and $x \in L_{-1}$, then $a x \in U$.

Proof. The proof of Lemma 41 is valid and shows that $a^{p}=0$. Hence $x R_{a}^{p}=0$. Suppose $x a$ has a nonzero $v$-component. Then $x R_{a}^{3}$ is a nonzero scalar multiple of $a^{\prime} a$. It follows that $a^{\prime} R_{a}^{p-2}=0$, a contradiction.

We are ready to handle the case of characteristic 2 . There is only one root space $L_{1}$. If $L_{1} v=0, v$ is central. Hence there exists $a$ in $L_{1}$ with $a^{\prime} \neq 0$. We shall prove $L_{1}^{2} \subset U$; this is a contradiction, for then $L^{2}=L$ fails to contain $v$. We have $a L_{1} \subset U$ by Lemma 78. Take any $b \in L_{1}$. If $b^{\prime} \neq 0$, then $b L_{1} \subset U$. If $b^{\prime}=0$, then $(a+b)^{\prime} \neq 0,(a+b) L_{1} \subset U, b L_{1} \subset U$.

The proof of Theorem 10 now continues only for the case of characteristic 3.

Lemma 79. For any $a \in L_{1}, a^{\prime} a=0$.

Proof. The argument is similar to the one just given. Assume the existence of $a \in L_{1}$ with $a^{\prime} a \neq 0$. Then $a L_{-1} \subset U$ by Lemma 78. Take any $b \in L_{1}$. If $b^{\prime} b \neq 0, b L_{-1} \subset U$. If $b^{\prime} b=0$, we study $c=a+b, d=a-b$. Since $c^{\prime} c+d^{\prime} d=-a^{\prime} a$, either $c^{\prime} c$ or $d^{\prime} d$ is nonzero, say $c^{\prime} c$. Then $(a+b) L_{-1} \subset U$, whence $b L_{-1} \subset U$. We have proved $L_{1} L_{-1} \subset U$, a contradiction for $L^{2}$ fails to contain $v$.

LemMa 80. $L_{1} v$ and $L_{-1} v$ are not 0 .

Proof. Assume $L_{-1} v=0$. Then $L_{1} v$ cannot be 0 ; otherwise $v$ would be central. From the Jacobi identity on $v, L_{1}, L_{-1}$ we get $L_{1} v \cdot L_{-1}=0$. Take $a$ in $L_{1}$ with $a^{\prime} \neq 0$. We have $a^{\prime} L_{-1}=0$ and by Lemma $79, a^{\prime} a=0$. From the Jacobi identity on $a, a^{\prime}, L_{-1}$ it then follows that $a L_{-1} \cdot a^{\prime}=0$, whence $a L_{-1} \subset V$, the one-dimensional subspace spanned by $v$. Take any $b \in L_{1}$. If $b^{\prime} \neq 0, b L_{-1} \subset V$ by what we have just shown. If $b^{\prime}=0$, then $(a+b)^{\prime} \neq 0,(a+b) L_{-1} \subset V$, $b L_{-1} \subset V$. Hence $L_{1} L_{-1} \subset V$, a contradiction since $u$ is not in the square of $L$.

Lemma 81. $R_{0}^{2}$ vanishes on $L_{1}$.

Proof. Assume the contrary. Then we have elements $a, b, c \neq 0$ in $L_{1}$ with $b=a^{\prime}, c=b^{\prime}$. Take any $x$ in $L_{-1}$ and apply the Jacobi identity to $a, b, x$. Since $a b=0$, we get $b x \cdot a+x a \cdot b=0$, and this equation requires that $a x$ lie in $U$. Take any $d$ in $L_{1}$. If $d R_{0}^{2} \neq 0$, then $d x \in U$ by what we just proved. If $d R_{0}^{2}=0$, then $(a+d) R_{0}^{2} \neq 0,(a+d) x \in U, d x \in U$. This shows that $L_{1} L_{-1} \subset U$, a contradiction.

We conclude the proof of Theorem 10 by producing an ideal in $L$, namely 
the subspace $S$ spanned by $v, M$, and $N$ where $M, N$ are the null spaces of $R_{v}$ in $L_{1}$ and $L_{-1}$. Of course, $S$ is invariant under $R_{u}$ and $R_{v}$. We have $L v \subset S$ by Lemma 81. What is left to prove is the following: for $c \in M$, show $c L_{1} \subset N$ and $c L_{-1} \subset V$. For the first we take any $a \in L_{1}$ and have to prove $(a c)^{\prime}=0$. Now $a^{\prime} a=(a+c)^{\prime}(a+c)=0$ by Lemma 79. Since $c^{\prime}=0$, we get $a^{\prime} c=0$, whence $(a c)^{\prime}=0$. Next take $x \in L_{-1}$. By Lemma 80 , there exists an element $b$ in $L_{1}$ with $b^{\prime} \neq 0$. We note that $b c \in N, b c \cdot x \in M$ by two applications of what we have just proved. Apply the Jacobi identity to $b, c, x$. The term $b x \cdot c$ lies in $M$. Hence so does $c x \cdot b$. This means $c x \in V$, as required.

We conclude this section with an example showing the failure of Theorem 6 for characteristic 3. (That Theorem 6 fails for characteristic 2 is quite evident; indeed in the case of characteristic 2 , one expects all roots to be isotropic. It should also be noted that Theorem 6 survives if the form is assumed to come from a restricted representation; the proof of Theorem 4.2 in [3] is valid.)

The example is 10-dimensional and has a 2-dimensional Cartan subalgebra with basis $u$, $v$. If we write $\alpha$ for the root which is 1 on $u, 0$ on $v$ and $\beta$ for the root which is 0 on $u, 1$ on $v$, the list of roots and corresponding root vectors reads: $\alpha, a ;-\alpha, b ; \beta, c ;-\beta, d ; \alpha+\beta, e ;-\alpha-\beta, f ; \alpha-\beta, g ; \beta-\alpha, h$. The products are as follows: $a c=b d=b e=c f=d e=0, a b=v, a d=g, a g=-f$, $a h=-c, b c=h, b f=-g, b g=-d, b h=e, c d=u, c e=g, c g=a, c h=-f, d f=-h$, $d g=e, d h=b, e f=u+v, e g=b, e h=d, f g=-c, f h=-a, g h=v-u$. The roots $\pm \alpha, \pm \beta$ are isotropic and the remaining ones nonisotropic. The form is given by $-f(u, v)=f(a, b)=f(c, d)=f(e, f)=f(g, h)=1$, with all other inner products vanishing. The algebra is simple and restricted.

15. Rank two, characteristic two. Without any assumption of an invariant form, we can give a complete classification of the simple restricted Lie algebras of rank two and characteristic two. It is not surprising that quadratic forms admitting composition play a role in the proof, for Lie algebras of characteristic two resemble Jordan algebras in many ways.

THEOREM 11. Let $L$ be a simple restricted Lie algebra of rank two over an algebraically closed field of characteristic two. Then the dimension of $L$ is 8,14 , or 26 , and in each case $L$ is uniquely determined.

By Theorem 10, a Cartan subalgebra $H$ of $L$ has basis $u, v$ satisfying $u^{2}=u, v^{2}=v$. There are (possibly) three root spaces which we label $A, B, C$; on $A, R_{u}$ is $1, R_{v}$ is 0 ; on $B, R_{u}$ is $0, R_{v}$ is 1 ; on $C, R_{u}$ and $R_{v}$ are both 1 .

Lemma 82. If $x \in A$ (resp. $B, C$ ) then $x^{2}$ is a scalar multiple of $v$ (resp. $u, u+v)$.

Proof. Assume $x \in A$. We have $x^{2} \in H$ and $x x^{2}=x R_{x}^{2}=0$. The only elements of $H$ annihilating a nonzero element of $A$ are the scalar multiples of $v$. The proof is similar if $x$ lies in $B$ or $C$. 
For any $x$ in $A$ we define $g(x)$ by the equation $x^{2}=g(x) v$. Manifestly $g$ is a quadratic form on $A$. We define $g$ similarly on $B$ and $C$. If $f$ is the corresponding bilinear form, then $x y=f(x, y) v$ for $x$ and $y$ in $A$; similarly for $B$ and $C$. There is no need for us to attempt to extend $g$ or $f$ beyond $A \cup B \cup C$.

Lemma 83. If $x$ and $y$ lie in $A \cup B \cup C$, but are not both in the same set, $g(x y)=g(x) g(y)$.

Proof. Assume for definiteness that $x$ lies in $A$ and $y$ in $B$. Then $x y$ lies in $C$ and $(x y)^{2}=g(x y)(u+v)$. To evaluate $g(x y)$ it suffices to test $(x y)^{2}$ on $x$. Now $x \cdot x y=y R_{x}^{2}=g(x) y ; y \cdot x y=x R_{y}^{2}=g(y) x$. Hence $x \cdot(x y)^{2}=g(x) g(y) x$. This shows that $g(x y)=g(x) g(y)$.

Lemma 84. If $a \in A, b \in B, c \in C$, then $f(a b, c)=f(b c, a)=f(c a, b)$.

Proof. By the Jacobi identity $a b \cdot c+b c \cdot a+c a \cdot b=0$. We thus have scalar multiples of $u+v, v$, and $u$ adding up to 0 . This is possible only if all three coefficients are equal.

LemMA 85. The form $f$ is nonsingular on $A$ (or $B$ or $C$ ).

Proof. Define $A_{0}$ to be the subset of $A$ annihilating $A$. Define $B_{0}, C_{0}$ similarly. We claim that $A_{0}+B_{0}+C_{0}$ is an ideal in $L$. Invariance under $R_{u}$ and $R_{v}$ is trivial. The typical thing that remains to be proved is $A_{0} B \subset C_{0}$, that is, $A_{0} B \cdot C=0$. Since $A_{0} \cdot B C=0$, this is immediate from Lemma 84 . By the simplicity of $L, A_{0}=0$, as required.

We now introduce a new multiplication in $A$. Fix elements $b \in B, c \in C$ with $g(b)=g(c)=1$. For $r, s \in A$ define $r * s=r b \cdot s c$.

Lemma 86. Under this multiplication $A$ has a two-sided unit element. Also, $g(r * s)=g(r) g(s)$.

Proof. The unit element is given by $a=b c$. For $a c=b R_{c}^{2}=b, r * a=r b \cdot a c$ $=r R_{b}^{2}=r$. Similarly, $a b=c, a * s=s$. That $g(r * s)=g(r) g(s)$ follows from repeated application of Lemma 83.

On $A$ we thus have a quadratic form admitting composition, in exactly the way this is formulated in [2]. Hence the dimension of $A$ is 2,4 , or 8 . Moreover the multiplication and quadratic form are uniquely determined in each case. The rest of the structure of $L$ can be reconstructed from this information. Three instances of this will suffice: (1) products $B B,(2)$ products $A B$, (3) products $B C$.

(1) Since $R_{c}$ maps $A$ onto $B$, a typical product in $B B$ has the form $r c \cdot s c(r, s \in A)$. By Lemma 84, $r c \cdot s c$ can be determined from $r(s c \cdot c)=r s$.

(2) A typical product in $A B$ has the form $r \cdot s c(r, s \in A)$. To identify $r \cdot s c$ it suffices to know its product with $b$ (since $R_{b}$ maps $C$ one-to-one onto $A)$. Now $(r \cdot s c) b=r b \cdot s c+r(s c \cdot b)=r * s+r(s c \cdot b)$. The scalar $s c \cdot b$ is known from (1). 
(3) A product in $B C$ is $r b \cdot s c$ which is simply $r * s$.

We remark finally that these three algebras have concrete realizations as follows: the 8-dimensional one is all 3 by 3 matrices of trace 0 ; the 14dimensional one is all 4 by 4 matrices of trace 0 , modulo scalars; the 26dimensional one is the cube $[[A A] A]$ of the algebra $A$ of 8 by 8 skewsymplectic matrices, modulo scalars.

Added in proof (September 5, 1958). By a supplement to Lemma 48, I have checked that Theorem 7 is also valid for characteristic 5 .

I take this opportunity to announce some further results. Assume characteristic at least 5 throughout.

1. If in a simple Lie algebra $L$ of rank one all root spaces are one-dimensional, then either $L$ is three-dimensional or the roots form a group.

2. In a simple restricted Lie algebra any three-dimensional Cartan subalgebra is abelian.

3. Assume that $L$ admits a nonsingular form and that the root space $L_{\alpha}$ is not one-dimensional. Then $\alpha$ is isotropic.

4. If in addition $L$ is simple then either all roots are nonisotropic (and $L$ is of the type classified by Seligman) or all are isotropic.

\section{BIBLIOGRAPHY}

1. N. Jacobson, Commutative restricted Lie algebras, Proc. Amer. Math. Soc. vol. 6 (1955) pp. $476-481$.

2. I. Kaplansky, Infinite-dimensional quadratic forms admitting composition, Proc. Amer. Math. Soc. vol. 4 (1953) pp. 956-960.

3. G. Seligman, On Lie algebras of prime characteristic, Memoirs Amer. Math. Soc., no. 19, New York, 1956.

4. H. Zassenhaus, Über Lie'sche Ringe mit Primzahlcharacteristik, Abh. Math. Sem. Univ. Hamburg vol. 13 (1939) pp. 1-100.

University of Chicago,

Chicago, Ill.

Princeton University,

Princeton, N. J. 